\title{
The Cartan form for constrained Lagrangian systems and the nonholonomic Noether theorem
}

\author{
M. Crampin and T. Mestdag \\ Department of Mathematics, Ghent University \\ Krijgslaan 281, S9, B-9000 Gent, Belgium
}

\begin{abstract}
This paper deals with conservation laws for mechanical systems with nonholonomic constraints. It uses a Lagrangian formulation of nonholonomic systems and a Cartan form approach. We present what we believe to be the most general relations between symmetries and first integrals. We discuss the so-called nonholonomic Noether theorem in terms of our formalism, and we give applications to Riemannian submanifolds, to Lagrangians of mechanical type, and to the determination of quadratic first integrals.
\end{abstract}

Mathematics Subject Classification (2000). 34A26, 37J60, 70G45, 70H03, 70H33.

Keywords. Lagrangian system, nonholonomic constraints, Noether theorem, symmetries, first integrals, conservation laws.

\section{Introduction}

This paper is concerned with some aspects of the search for conservation laws for mechanical systems with nonholonomic constraints (i.e. velocity-dependent constraints). Such constraints show up naturally e.g. in robotics and in control theory when one considers rigid bodies rolling over a surface or over each other, or possessing a contact point with a surface in the form of a knife edge. Standard references to the differential geometric approach to systems with nonholonomic constraints are the recent books [2, 7, 14]. In this paper we follow a Lagrangian formulation of nonholonomic systems. The constraints will be assumed to be linear in velocities and both the Lagrangian and the constraints will be assumed to be independent of time.

We shall investigate the relation between first integrals (or constants of the motion) on the one hand and symmetries on the other hand. In the absence of nonholonomic constraints, this relation is often described by what is commonly called 'Noether's (first) theorem'. Although anyone working in the field is familiar with this terminology (see [27] for a recent book on the history of the subject), a quick scan through the literature immediately reveals that the precise formulation of the theorem is somewhat subject to personal taste. In fact, the terminology 'Noether's theorem' is associated with many slightly different manifestations of the same group of underlying ideas. For example, some authors relate Noether's theorem to invariance transformations of the action functional (up to a gauge term) depending on position (and possibly time) only, while others also use the term Noether's theorem for generalizations to velocity-dependent transformations. At the infinitesimal level, the first point of view relates to the existence of a vector field on the configuration space whose complete or tangent lift to velocity space preserves 
the Lagrangian, while the second viewpoint is related to the existence of a vector field on velocity space, not necessarily projectable to one on the configuration space, that, among other things, preserves the so-called Cartan 2-form. Throughout this paper, we shall refer only to the first viewpoint as the 'Noether theorem', while we shall call the second viewpoint the 'Cartan form approach'. For unconstrained Lagrangian systems, the relation between the two viewpoints was well established in e.g. [9], where it is argued that the Cartan form approach is superior since it embodies a simple direct correspondence between symmetries and first integrals, and in any case contains the Noether theorem (in the first sense) as a special case. Besides the two above-mentioned versions of the Noether theorem, one may find in the literature many other generalizations. For a review on those we refer to [32].

Given the lack of consensus for standard Lagrangian systems, it is no surprise that the situation is even more troublesome for the translation of the ideas behind the Noether theorem to the context of Lagrangian systems with additional nonholonomic constraints. It is well-known that for those systems the relation between symmetries and constants of the motion is no longer as natural as it is for the unconstrained case. Nevertheless, many people have investigated the conditions under which symmetry properties of the system do lead to conservation laws. These investigations have resulted mostly in generalizations of the two viewpoints described above for the unconstrained situation. For example, papers discussing the first point of view are those by Fassò and co-workers [19, 20, 21, 22] and Iliev et al. [24, 25]. Results concerning the Cartan form viewpoint may be found in e.g. [23] (for time-dependent systems) and [1, 8, 15] (from the Hamiltonian point of view). Actually, the bulk of the literature focusses on a very special case: the one where the system is invariant under the tangent lift of an action of a Lie group (see e.g. [3, 4, 5, 6, 26, 33]) (none of these lists is meant to be exhaustive). Unfortunately, for nonholonomic systems it is far from immediately obvious how the two distinct viewpoints are to be compared. The main goal of this paper is to come to a transparent description of these two levels of generalization and of their interaction.

Throughout the paper we shall take advantage of a simple and well-known observation we have used also in previous publications [11, 12]: the dynamics can be represented by means of a vector field, and so can any symmetry (at an infinitesimal level). In Section 2 we recall the version of the d'Alembert principle we stated in [11] and we interpret it here in terms of the fibre metric given by the Hessian of the Lagrangian. In the following section we discuss some generalities concerning the restriction of the Cartan 2-form to the constraint submanifold, we present what we believe to be the most general relations between symmetries and constants of the motion in the Cartan form approach and we discuss the special case where the nonholonomic distribution is maximally non-integrable. In the fourth section we translate the nonholonomic Noether theorem of [21] to our formalism. We further show how it can be derived from the results in the previous section and we discuss some special cases. The last section contains applications of the previous results to Riemannian submanifolds, to Lagrangians of mechanical type, and to the search for linear and quadratic integrals.

We shall assume that the reader is familiar with the basic tools and concepts needed for the geometric description of Lagrangian systems, such as the vertical and complete lifts $X^{\mathrm{V}}$ and $X^{\mathrm{C}}$ of a vector field $X$ on $Q$, the vertical endomorphism $S$, the concept of a second-order ordinary differential equation vector field, etc. For definitions and basic properties we refer to e.g. [13, 17]. 


\section{The Lagrange-d'Alembert principle and the fibre metric}

Our starting point is the formulation of the equations determining the dynamics of a regular Lagrangian system subject to nonholonomic linear constraints which we gave in [11. The constraints may be defined by either a distribution $\mathcal{D}$ on configuration space $Q$ (the constraint distribution), or a submanifold $\mathcal{C}$ of $T Q$ (the constraint submanifold). The two are related as follows: $\mathcal{C}=\left\{u \in T Q: u \in \mathcal{D}_{q} \subset T_{q} Q, q=\tau(u)\right\} ; \tau$ stands here for the tangent bundle projection $T Q \rightarrow Q$. We assume that the dimension of each $\mathcal{D}_{q}$ is constant and equal to $m$. Throughout the paper we shall denote by $\iota$ the injection $\mathcal{C} \rightarrow T Q$. A vector field $\Gamma$ on $\mathcal{C}$ is said to be of second-order type if it satisfies $\tau_{* u} \Gamma=u$ for all $u \in \mathcal{C}$. A Lagrangian function $L$ on $T Q$ is said to be regular with respect to $\mathcal{D}$ if for any local basis $\left\{X_{\alpha}\right\}$ of $\mathcal{D}, 1 \leq \alpha \leq m$, the symmetric $m \times m$ matrix whose entries are $X_{\alpha}^{\mathrm{V}}\left(X_{\beta}^{\mathrm{V}}(L)\right)$ (functions on $\mathcal{C}$ ) is nonsingular. In [11] we proved the following proposition.

Proposition 1. Let $L$ be a Lagrangian on $T Q$ which is regular with respect to $\mathcal{D}$. Then there is a unique vector field $\Gamma$ on $\mathcal{C}$ which is of second-order type, is tangent to $\mathcal{C}$, and is such that on $\mathcal{C}$

$$
\Gamma\left(Z^{\mathrm{V}}(L)\right)-Z^{\mathrm{C}}(L)=0
$$

for all $Z \in \mathcal{D}$. Moreover, $\Gamma$ may be determined from the equations

$$
\Gamma\left(X_{\alpha}^{\mathrm{V}}(L)\right)-X_{\alpha}^{\mathrm{C}}(L)=0, \quad \alpha=1,2, \ldots m,
$$

on $\mathcal{C}$, where $\left\{X_{\alpha}\right\}$ is any local basis for $\mathcal{D}$.

This is our version of the Lagrange-d'Alembert principle; the vector field $\Gamma$ is the dynamical field of the constrained system.

We shall make use of the following result. For any smooth map between manifolds $\phi: M \rightarrow N$, if $\xi \in \mathfrak{X}(M)$ (the module of smooth vector fields on $M$ ) and $\eta \in \mathfrak{X}(N)$ are $\phi$-related and $\chi$ is any form on $N$ then $\mathcal{L}_{\xi}\left(\phi^{*} \chi\right)=\phi^{*}\left(\mathcal{L}_{\eta} \chi\right)$. We shall apply it with $\phi=\iota$, when $\xi$ is tangent to $\mathcal{C}$ (so that the restriction of $\xi$ to $\mathcal{C}$ is $\iota$-related to $\xi$ itself), to obtain $\mathcal{L}_{\xi}\left(\iota^{*} \chi\right)=\iota^{*}\left(\mathcal{L}_{\xi} \chi\right)$ (where on the left-hand side $\xi$ should be understood as $\left.\xi\right|_{\mathcal{C}}$ ). This holds, mutatis mutandis, when $\chi$ is a function. In a similar vein, if $\xi$ is tangent to $\mathcal{C}$ then $\left.\xi\lrcorner \iota^{*} \chi=\iota^{*}(\xi\lrcorner \chi\right)$.

We shall assume that the Lagrangian $L$, in addition to being regular with respect to $\mathcal{D}$, is also regular with respect to $T Q$ (i.e. that $L$ is a regular Lagrangian in the standard sense). Remark that in case the Hessian of $L$ is positive definite, $L$ will automatically be regular with respect to both $T Q$ and $\mathcal{D}$. The dynamical vector field $\Gamma$ defined in the proposition above should not be confused with the standard Euler-Lagrange field $\Gamma_{0}$ of $L$ on $T Q$. The latter is uniquely determined by the condition that on $T Q$ (i.e. not only on $\mathcal{C}$ )

$$
\Gamma_{0}\left(Z^{\mathrm{V}}(L)\right)-Z^{\mathrm{C}}(L)=0
$$

for all vector fields on $Q$ (i.e. not only those in $\mathcal{D}$ ). We shall not make a notational distinction between $\Gamma_{0}$ and its restriction to $\mathcal{C}$. It is easy to see that, on $\mathcal{C}, \Gamma-\Gamma_{0}$ is vertical (with respect to the projection $\tau$ ).

Let $g$ be the Hessian of $L$ with respect to fibre coordinates. It can be considered as a fibre metric on $T Q$, so that it defines a scalar product of vertical vectors, as follows. Let us equip each fibre of $T Q$, which is of course a vector space, with the flat connection, and denote its covariant derivative 
operator by $\nabla^{0}$. Then for any pair of vertical vector fields $V$ and $W, \nabla_{V}^{0} W$ makes sense, and is a vertical vector field. Moreover $\nabla_{V}^{0} W-\nabla_{W}^{0} V=[V, W]$. Set $g(V, W)=V(W(L))-\nabla_{V}^{0} W(L)$. Then $g$ is bilinear over $C^{\infty}(T Q)$ and symmetric. If we take $V=X^{\mathrm{v}}, W=Y^{\mathrm{v}}$ for vector fields $X, Y$ on $Q$ we get back the usual definition of the Hessian, $X^{\mathrm{V}}\left(Y^{\mathrm{v}}(L)\right)=g\left(X^{\mathrm{v}}, Y^{\mathrm{v}}\right)$, since $\nabla_{V}^{0} Y^{\mathrm{V}}=0$ for any vertical $V$; but note that we have extended the ring of coefficients from $C^{\infty}(Q)$ to $C^{\infty}(T Q)$.

The interesting point is that $\Gamma-\Gamma_{0}$ is perpendicular (with respect to $g$ ) to $X^{\mathrm{V}}$ for all $X$ in $\mathcal{D}$. So in an obvious sense, $\Gamma$ is the perpendicular projection of the unconstrained dynamics $\Gamma_{0}$ onto $\mathcal{C}$. The basic fact is evident: on $\mathcal{C},\left(\Gamma-\Gamma_{0}\right)\left(X^{\mathrm{V}}(L)\right)=0$ for all $X$ in $\mathcal{D}$, since $\left.\Gamma\left(X^{\mathrm{V}}(L)\right)=X^{\mathrm{C}}(L)\right)=$ $\Gamma_{0}\left(X^{\mathrm{V}}(L)\right)$. In view of the definition of $g$ it follows that $g\left(\Gamma-\Gamma_{0}, X^{\mathrm{V}}\right)=0$ for all $X \in \mathcal{D}$. In fact $g\left(\Gamma-\Gamma_{0}, V\right)=0$ for any vertical vector field $V$ tangent to $\mathcal{C}$; or if we restrict attention to any fibre $T_{q} Q$, we see that $\left(\Gamma-\Gamma_{0}\right)_{T_{q} Q}$ is normal, with respect to $\left.g\right|_{T_{q} Q}$, to the submanifold (indeed, linear subspace) $\mathcal{C}_{q}$ of $T_{q} Q$. It will be convenient to say that a vector field $V$ is fibre-normal to $\mathcal{C}$ if it is vertical and $g(V, W)=0$ for all vertical vector fields $W$ which are tangent to $\mathcal{C}$. Then $\Gamma-\Gamma_{0}$ is fibre-normal to $\mathcal{C}$.

This discussion may be summarized in the form of the following proposition, which amounts to an alternative formulation of the Lagrange-d'Alembert principle of Proposition 1 .

Proposition 2. Let $L$ be a Lagrangian on $T Q$ which is regular with respect to both $\mathcal{D}$ and $T Q$. Then the dynamical field $\Gamma$ of the corresponding constrained system is the unique vector field tangent to $\mathcal{C}$ such that $\Gamma-\Gamma_{0}$ is vertical and fibre-normal to $\mathcal{C}$ with respect to the fibre metric determined by $L$.

Note that $\Gamma$ is necessarily of second-order type since it differs from a second-order differential equation field by a vertical field.

In what follows we shall always implicitly assume that $L$ is regular with respect to both $\mathcal{D}$ and $T Q$.

The fact that $\Gamma$ is the image of $\Gamma_{0}$ under a projection operator appears in a paper of de León and Martín de Diego [16], but the result is derived there in a way which somewhat obscures the role of the Hessian $g$.

\section{The Cartan form approach}

Let $S$ be the vertical endomorphism on $T Q$. It can be defined by means of its action on vertical and complete lifts: $S\left(X^{\mathrm{C}}\right)=X^{\mathrm{V}}$ and $S\left(X^{\mathrm{V}}\right)=0$. It is common to call the form $\theta_{L}=d L \circ S$, or more succinctly $S(d L)$, the Cartan 1-form; the Cartan 2-form is $\omega_{L}=d \theta_{L}$. Some authors also use 'Poincaré-Cartan forms' or simply 'Poincaré forms' for these forms. According to [28] Poincaré was the first to introduce the forms, while Cartan defined their extension to the context of timedependent Lagrangians. The Cartan 2-form can be thought of as the Kähler lift of the Hessian $g$. As such, $\omega_{L}$ evaluated on a pair of vertical vectors gives zero, $\omega_{L}(S(X), Y)=\omega_{L}(S(Y), X)$, and if $V$ is vertical then $\omega_{L}(V, Z)=g(V, S(Z))$. The next subsection is concerned with the properties of the restriction of the form $\omega_{L}$ to the constraint submanifold $\mathcal{C}$, in both senses: $\left.\omega_{L}\right|_{\mathcal{C}}$ and $\iota^{*} \omega_{L}$.

At any point $u \in \mathcal{C} \subset T Q$, the tangent space $T_{u} \mathcal{C}$ projects onto $T_{q} Q$ (where $q=\tau(u)$ ), and the kernel of the projection is the vertical lift of $\mathcal{D}_{q}$. We denote the vertical lift of $\mathcal{D}_{q}$ to $u$ by $\mathcal{D}_{u}^{\mathrm{V}}$, 
and the vertical subspace of $T_{u}(T Q)$ by $V_{u}(T Q)$. The subspace of $V_{u}(T Q)$ consisting of vectors fibre-normal to $\mathcal{D}_{u}^{\mathrm{V}}$, say $\left(\mathcal{D}_{u}^{\mathrm{V}}\right)^{\perp}$, is complementary to $T_{u} \mathcal{C}$ in $T_{u}(T Q)$. An obvious move is to consider those vectors $\xi \in T_{u} \mathcal{C}$ such that $S(\xi) \in \mathcal{D}_{u}^{\mathrm{V}}$, and those such that $S(\xi) \in\left(\mathcal{D}_{u}^{\mathrm{V}}\right)^{\perp}$. Denote the former by $\tilde{\mathcal{D}}_{u}$, the latter by $\tilde{\mathcal{D}}_{u}^{\perp}$. Then $\tilde{\mathcal{D}}_{u}$ and $\tilde{\mathcal{D}}_{u}^{\perp}$ are subspaces of $T_{u} \mathcal{C}$, which together span it; but they are not complementary, in fact $\tilde{\mathcal{D}}_{u} \cap \tilde{\mathcal{D}}_{u}^{\perp}=\mathcal{D}_{u}^{\mathrm{V}}$.

Let us consider the distribution $\tilde{\mathcal{D}}: u \mapsto \tilde{\mathcal{D}}_{u}$ in greater detail. It is clearly projectable to $Q$, and $\tau_{\mid \mathcal{C} *} \tilde{\mathcal{D}}=\mathcal{D}$. Its kernel under projection is just $\mathcal{D}^{\mathrm{V}}$, that is, the vertical distribution on $\mathcal{C}$. So the fact that $\tau_{\mid \mathcal{C} *} \tilde{\mathcal{D}}=\mathcal{D}$ defines $\tilde{\mathcal{D}}$; we can write $\tilde{\mathcal{D}}_{u}=\tau_{\mid \mathcal{C} * u}{ }^{-1}\left(\mathcal{D}_{\tau(u)}\right)$.

We can now use the distributions $\tilde{\mathcal{D}}$ and $\tilde{\mathcal{D}}^{\perp}$ to construct a convenient local basis for $\mathfrak{X}(T Q)$, or in other words an anholonomic frame on $T Q$, adapted to the study of the restriction of $\omega_{L}$ to $\mathcal{C}$.

\subsection{Construction of a suitable anholonomic frame}

We can evidently find local vector fields $\mathcal{X}_{\alpha} \in \tilde{\mathcal{D}}$ and $\mathcal{X}_{a} \in \tilde{\mathcal{D}}^{\perp}$ such that

- for each $u,\left\{\tau_{* u} \mathcal{X}_{\alpha}, \tau_{* u} \mathcal{X}_{a}\right\}$ is a basis for $T_{q} Q$;

- $\left\{S\left(\mathcal{X}_{\alpha}\right)\right\}$ is a basis for $\mathcal{D}^{\mathrm{v}}$;

- $\left\{S\left(\mathcal{X}_{a}\right)\right\}$ is a basis for $\left(\mathcal{D}^{\mathrm{V}}\right)^{\perp}$.

We could start with a basis $\left\{X_{i}\right\}=\left\{X_{\alpha}, X_{a}\right\}$ of vector fields on $Q$ (an anholonomic frame on $Q$ ) with $\left\{X_{\alpha}\right\}$ a basis for $\mathcal{D}$. Take for $\mathcal{X}_{\alpha}$ some projection of $X_{\alpha}^{\mathrm{C}}$ into $T \mathcal{C}$ along vertical vectors: then $S\left(\mathcal{X}_{\alpha}\right)=X_{\alpha}^{\mathrm{V}}$. By suitable similar modifications of the $X_{a}^{\mathrm{C}}$ we can fulfill the other requirements. Note that the vector fields $\mathcal{X}_{i}$ cannot be assumed to be projectable to $Q$.

Denote $S\left(\mathcal{X}_{\alpha}\right)$ by $\mathcal{Y}_{\alpha}$ and $S\left(\mathcal{X}_{a}\right)$ by $\mathcal{Y}_{a}$. Then $\left\{\mathcal{X}_{\alpha}, \mathcal{X}_{a}, \mathcal{Y}_{\alpha}\right\}$ is a local basis for $\mathfrak{X}(\mathcal{C})$, and $\left\{\mathcal{X}_{\alpha}, \mathcal{X}_{a}, \mathcal{Y}_{\alpha}, \mathcal{Y}_{a}\right\}$ is a local basis for $\mathfrak{X}(T Q)$. We have $g\left(\mathcal{Y}_{\alpha}, \mathcal{Y}_{a}\right)=0$, where $g$ is the fibre metric. Let us set $g\left(\mathcal{Y}_{\alpha}, \mathcal{Y}_{\beta}\right)=g_{\alpha \beta}$ and $g\left(\mathcal{Y}_{a}, \mathcal{Y}_{b}\right)=g_{a b}$. If $L$ is regular with respect to $\mathcal{D},\left(g_{\alpha \beta}\right)$ is nonsingular. From the above relation between $\omega_{L}$ and $g$, we get

$$
\omega_{L}\left(\mathcal{Y}_{\alpha}, \mathcal{X}_{\beta}\right)=g_{\alpha \beta}, \quad \omega_{L}\left(\mathcal{Y}_{a}, \mathcal{X}_{b}\right)=g_{a b}, \quad \omega_{L}\left(\mathcal{Y}_{\alpha}, \mathcal{X}_{a}\right)=\omega_{L}\left(\mathcal{Y}_{a}, \mathcal{X}_{\alpha}\right)=0
$$

Set $\omega_{L}\left(\mathcal{X}_{\alpha}, \mathcal{X}_{\beta}\right)=\omega_{\alpha \beta}$ and so on. If we change $\mathcal{X}_{a}$ to $\overline{\mathcal{X}}_{a}=\mathcal{X}_{a}-g^{\alpha \beta} \omega_{a \beta} \mathcal{Y}_{\alpha}$ then $\overline{\mathcal{X}}_{a} \in \tilde{\mathcal{D}}_{u}^{\perp}$, $S\left(\overline{\mathcal{X}}_{a}\right)=\mathcal{Y}_{a}$, but

$$
\omega_{L}\left(\overline{\mathcal{X}}_{a}, \mathcal{X}_{\alpha}\right)=\omega_{a \alpha}-g^{\beta \gamma} \omega_{a \beta} g_{\alpha \gamma}=0 .
$$

So without loss of generality we can assume that $\omega_{a \alpha}=0$. We are still free to modify $\mathcal{X}_{\alpha}$ similarly, by the addition of a linear combination of the $\mathcal{Y}_{\alpha}$. Since $g_{a \alpha}=0$, this won't alter the value of $\omega_{a \alpha}$. Let $\overline{\mathcal{X}}_{\alpha}=\mathcal{X}_{\alpha}-\frac{1}{2} g^{\beta \gamma} \omega_{\alpha \gamma} \mathcal{Y}_{\beta}$. Then

$$
\omega_{L}\left(\overline{\mathcal{X}}_{\alpha}, \overline{\mathcal{X}}_{\beta}\right)=\omega_{\alpha \beta}-\frac{1}{2} g^{\gamma \delta} \omega_{\alpha \delta} g_{\beta \gamma}+\frac{1}{2} g^{\gamma \delta} \omega_{\beta \delta} g_{\alpha \gamma}=0 .
$$

So without loss of generality we can further assume that $\omega_{\alpha \beta}=0$.

We shall assume from now on that these modifications have been made, and we shall drop the overbars. The constructions of the previous paragraphs may then be summarized as follows.

Consider the following three distributions on $\mathcal{C}$.

- $\mathcal{D}^{\mathrm{V}}=\left\langle\mathcal{Y}_{\alpha}\right\rangle$, of dimension $\operatorname{dim} \mathcal{D}=m$, is the vertical lift of $\mathcal{D}$ to $\mathcal{C}$; 
- $\hat{\mathcal{D}}=\left\langle\mathcal{X}_{\alpha}\right\rangle$, of dimension $m$, projects onto $\mathcal{D}$, satisfies $S(\hat{\mathcal{D}})=\mathcal{D}^{\mathrm{v}}$, is a complement to $\mathcal{D}^{\mathrm{v}}$ in $\tilde{\mathcal{D}}$, and is isotropic with respect to $\omega_{L}$;

- $\tilde{\mathcal{D}}^{\top}=\left\langle\mathcal{X}_{a}\right\rangle$, of dimension $n-m$, projects onto a complement to $\mathcal{D}$ in $\mathfrak{X}(Q)$, is a complement to $\tilde{\mathcal{D}}$ in $\mathfrak{X}(\mathcal{C})$, and is symplectically orthogonal to $\tilde{\mathcal{D}}$; it satisfies $S\left(\tilde{\mathcal{D}}^{\top}\right)=\left(\mathcal{D}^{\mathrm{V}}\right)^{\perp}$.

These three distributions are related to $\tilde{\mathcal{D}}$, to $\mathfrak{X}(\mathcal{C})$ and to $\mathfrak{X}(T Q)$ as follows:

$$
\begin{aligned}
\tilde{\mathcal{D}} & =\mathcal{D}^{\mathrm{V}} \oplus \hat{\mathcal{D}} \\
\mathfrak{X}(\mathcal{C}) & =\tilde{\mathcal{D}} \oplus \tilde{\mathcal{D}}^{\top} \\
\mathfrak{X}(T Q) & =\mathfrak{X}(\mathcal{C}) \oplus\left(\mathcal{D}^{\mathrm{V}}\right)^{\perp} .
\end{aligned}
$$

Let $\left\{\vartheta^{\alpha}, \vartheta^{a}, \varphi^{\alpha}, \varphi^{a}\right\}$ be the local basis of 1-forms on $T Q$, or coframe, which is dual to the basis $\left\{\mathcal{X}_{\alpha}, \mathcal{X}_{a}, \mathcal{Y}_{\alpha}, \mathcal{Y}_{a}\right\}$ of $\mathfrak{X}(T Q)$. Then $\vartheta^{i}=S\left(\varphi^{i}\right)(i=\alpha, a)$. Also, $\left\{\vartheta^{\alpha}, \vartheta^{a}, \varphi^{\alpha}\right\}$, when restricted to acting on $\mathfrak{X}(\mathcal{C})$, is a local basis of 1 -forms on $\mathcal{C}$ (or more accurately, $\left\{\iota^{*} \vartheta^{\alpha}, \iota^{*} \vartheta^{a}, \iota^{*} \varphi^{\alpha}\right\}$ is a basis for 1 -forms on $\mathcal{C}$; however, we shall usually ignore this refinement, trusting that it will be clear from the context what is intended); and $\left\langle\varphi^{a}\right\rangle$ is $\mathfrak{X}(\mathcal{C})^{\circ}$, the annihilator of $\mathfrak{X}(\mathcal{C})$, so that $\iota^{*} \varphi^{a}=0$. In terms of this coframe

$$
\left.\omega_{L}\right|_{\mathcal{C}}=g_{\alpha \beta} \varphi^{\alpha} \wedge \vartheta^{\beta}+g_{a b} \varphi^{a} \wedge \vartheta^{b}+\frac{1}{2} \omega_{a b} \vartheta^{a} \wedge \vartheta^{b} .
$$

Evidently for $\left.\omega_{L}\right|_{\mathcal{C}}$ to be symplectic (that is, for $L$ to be regular) it must be the case that $\left(g_{a b}\right)$ is nonsingular (this will automatically be so if the Hessian of $L$ is positive definite). Assuming $L$ is regular, at any $u \in \mathcal{C}$ the symplectic orthogonal to $T_{u} \mathcal{C}$ in $T_{u}(T Q)$ is given by

$$
\left(T_{u} \mathcal{C}\right)^{\top}=\left\langle\mathcal{X}_{a}+g^{b c} \omega_{a b} \mathcal{Y}_{c}\right\rangle_{u}
$$

The characteristic subspace $\chi\left(\iota^{*} \omega_{L}\right)$ of $\iota^{*} \omega_{L}$ is $\left(T_{u} \mathcal{C}\right)^{\top} \cap T_{u} \mathcal{C}$, and is therefore given by

$$
\chi\left(\iota^{*} \omega_{L}\right)=\left\{\xi^{a} \mathcal{X}_{a}: \xi^{b} \omega_{a b}=0\right\}=\chi\left(\left.\omega_{L}\right|_{\tilde{\mathcal{D}}_{u}^{\top}}\right) .
$$

In summary, we have the following proposition.

Proposition 3. We can find a frame $\left\{\mathcal{X}_{\alpha}, \mathcal{X}_{a}, \mathcal{Y}_{\alpha}, \mathcal{Y}_{a}\right\}$ on $T Q$ such that $\left\langle\mathcal{Y}_{\alpha}\right\rangle=\mathcal{D}^{\mathrm{V}},\left\langle\mathcal{X}_{\alpha}, \mathcal{Y}_{\alpha}\right\rangle=$ $\tilde{\mathcal{D}},\left\langle\mathcal{X}_{\alpha}, \mathcal{X}_{a}, \mathcal{Y}_{\alpha}\right\rangle=\mathfrak{X}(\mathcal{C}),\left\langle\mathcal{Y}_{a}\right\rangle=\left(\mathcal{D}^{\mathrm{V}}\right)^{\perp}$; and such that with respect to the dual coframe $\left\{\vartheta^{\alpha}, \vartheta^{a}, \varphi^{\alpha}, \varphi^{a}\right\}$ we have $\left\langle\varphi^{a}\right\rangle=\mathfrak{X}(\mathcal{C})^{\circ}$ and

$$
\iota^{*} \omega_{L}=g_{\alpha \beta} \varphi^{\alpha} \wedge \vartheta^{\beta}+\frac{1}{2} \omega_{a b} \vartheta^{a} \wedge \vartheta^{b}
$$

(Strictly speaking there should be an $\iota^{*}$ on each 1 -form on the right-hand side of the final formula.) We shall use this expression for $\iota^{*} \omega_{L}$ repeatedly below. Note that at $u \in \mathcal{C}$ the first term on the right-hand side is the restriction of $\iota^{*} \omega_{L}$ to $\tilde{\mathcal{D}}_{u}$, the second its restriction to $\tilde{\mathcal{D}}_{u}^{\top}$; these are complementary subspaces of $T_{u} \mathcal{C}$ and are symplectically orthogonal. Moreover, $\chi\left(\iota^{*} \omega_{L}\right) \subset \tilde{\mathcal{D}}_{u}^{\top}$; as is evident also from the expression above, $\iota^{*} \omega_{L}$ is nonsingular on $\tilde{\mathcal{D}}_{u}$.

Of course, we do not claim that the frame described in the proposition is unique. 


\subsection{First integrals and symmetries}

We are now ready to establish some relations between first integrals on the one hand and symmetries on the other. Some of the results in this section are closely related to analogous results that may be found in [23] (albeit in the context of time-dependent constraints) and [1, 15] (albeit in a Hamiltonian formalism). The reader should also keep in mind that by setting the constraint submanifold $\mathcal{C}$ equal to the whole tangent manifold $T Q$, one recovers the well-known relations between symmetries and constants of motion, to be found in e.g. [9, 32,

Let us now work locally on $\mathcal{C}$, in terms of the local bases of vector fields $\left\{\mathcal{X}_{\alpha}, \mathcal{Y}_{\alpha}, \mathcal{X}_{a}\right\}$, and the dual basis of 1 -forms (i.e. sections of $\left.T^{*} \mathcal{C} \rightarrow \mathcal{C}\right)\left\{\vartheta^{\alpha}, \varphi^{\alpha}, \vartheta^{a}\right\}$. Note that $\left\{\vartheta^{a}\right\}$ is a basis for $\tilde{\mathcal{D}}^{\circ}$, the annihilator of $\tilde{\mathcal{D}}$.

Given a 1 -form $\psi$ on $\mathcal{C}$, there need not be a vector field $Z$ on $\mathcal{C}$ such that $Z\lrcorner \iota^{*} \omega_{L}=\psi$ : it is necessary that $\psi(W)=0$ for all $W \in \chi\left(\iota^{*} \omega_{L}\right)$; and if such $Z$ exists it won't be unique. However, we have the following important result, which establishes a modified construction for obtaining vector fields from 1-forms.

Proposition 4. For any 1 -form $\psi$ on $\mathcal{C}$, there is a unique vector field $Z$ on $\mathcal{C}$ such that $Z \in \tilde{\mathcal{D}}$ and $Z\lrcorner \iota^{*} \omega_{L}-\psi \in \tilde{\mathcal{D}}^{\circ}$.

Proof. The vector field $Z$ given by

$$
Z=g^{\alpha \beta}\left(\left\langle\mathcal{X}_{\beta}, \psi\right\rangle \mathcal{Y}_{\alpha}-\left\langle\mathcal{Y}_{\beta}, \psi\right\rangle \mathcal{X}_{\alpha}\right)
$$

is well defined because of the assumed regularity of $L$. It belongs to $\tilde{\mathcal{D}}$ and satisfies $Z\lrcorner \iota^{*} \omega_{L}-\psi \in$ $\tilde{\mathcal{D}}^{\circ}$. Now if $Y \in \tilde{\mathcal{D}}$ then $\left.Y\right\lrcorner \iota^{*} \omega_{L} \in\left\langle\vartheta^{\alpha}, \varphi^{\alpha}\right\rangle$, by inspection of the expression for $\iota^{*} \omega_{L} ;$ so if $Y \in \tilde{\mathcal{D}}$ and $Y\lrcorner \iota^{*} \omega_{L} \in \tilde{\mathcal{D}}^{\circ}$ then $Y=0$, and the vector field $Z$ displayed above is uniquely determined.

The penultimate statement is worth recording separately.

Corollary 1. If $Z \in \tilde{\mathcal{D}}$ and $Z\lrcorner \iota^{*} \omega_{L} \in \tilde{\mathcal{D}}^{\circ}$ then $Z=0$.

It is well-known (see [13, 17]) that $\Gamma_{0}$, the dynamical vector field of the unconstrained system, can be characterized as the unique second-order vector field satisfying $\left.\Gamma_{0}\right\lrcorner \omega_{L}+d E_{L}=0$. The function $E_{L}=\Delta(L)-L$ is the energy of the Lagrangian $L$ and $\Delta$ is the Liouville field (the infintesimal generator of scaling transformations). It follows that the dynamical vector field of the constrained system, $\Gamma$, is determined by the 1 -form $-d\left(\iota^{*} E_{L}\right)$ in the way described in Proposition 4. Recall first of all that $\Gamma \in \tilde{\mathcal{D}}$ (it is tangent to $\mathcal{C}$ and at each $u \in \mathcal{C}, \tau_{* u} \Gamma=u \in$ $\left.\mathcal{D}_{\tau(u)}\right)$; and secondly that $\Gamma-\Gamma_{0} \in\left(\mathcal{D}^{\mathrm{V}}\right)^{\perp}$ : say $\Gamma=\Gamma_{0}+\gamma^{a} \mathcal{Y}_{a}$ (the $\gamma^{a}$ are multipliers in some manifestation). Then on $\mathcal{C}$

$$
\left.\left.\Gamma\lrcorner \omega_{L}+d E_{L}=\left(\Gamma-\Gamma_{0}\right)\right\lrcorner \omega_{L}=\gamma^{a} \mathcal{Y}_{a}\right\lrcorner \omega_{L}=\gamma^{b} g_{a b} \vartheta^{a} .
$$

Now apply $\iota^{*}$, and the result follows.

Theorem 1. For $f$ a function on $\mathcal{C}$, let $Z_{f}$ be the unique vector field on $\mathcal{C}$ such that $Z_{f} \in \tilde{\mathcal{D}}$ and $\left.Z_{f}\right\lrcorner \iota^{*} \omega_{L}-d f \in \tilde{\mathcal{D}}^{\circ}$. Then $f$ is a first integral of $\Gamma$ if and only if $Z_{f}\left(\iota^{*} E_{L}\right)=0$. 
Proof. Since $\Gamma, Z_{f} \in \tilde{\mathcal{D}}$

$$
\left.\left.\left.\Gamma(f)=\Gamma\lrcorner\left(Z_{f}\right\lrcorner \iota^{*} \omega_{L}\right)=-Z_{f}\right\lrcorner(\Gamma\lrcorner \iota^{*} \omega_{L}\right)=Z_{f}\left(\iota^{*} E_{L}\right),
$$

and the result follows.

This procedure sets up a 1-1 correspondence between local first integrals of $\Gamma$ (determined up to the addition of a constant) and vector fields $Z \in \tilde{\mathcal{D}}$ such that $\mathcal{L}_{Z}\left(\iota^{*} \omega_{L}\right) \in d\left(\tilde{\mathcal{D}}^{\circ}\right)$ (i.e. such that $\mathcal{L}_{Z}\left(\iota^{*} \omega_{L}\right)=d \phi$ for some $\left.\phi \in \tilde{\mathcal{D}}^{\circ}\right)$ and $Z\left(\iota^{*} E_{L}\right)=0$. Indeed, $\left.\mathcal{L}_{Z}\left(\iota^{*} \omega_{L}\right)=d(Z\lrcorner \iota^{*} \omega_{L}\right)=d \phi$ for $\phi \in \tilde{\mathcal{D}}^{\circ}$ if and only if $\left.Z\right\lrcorner \iota^{*} \omega_{L}$ differs from $\phi \in \tilde{\mathcal{D}}^{\circ}$ by a closed, and so locally exact, 1-form. If we set $\mathcal{L}_{Z}\left(\iota^{*} \theta_{L}\right)-\phi=d F$, then $f$ above is given by $f=F-\iota^{*} \theta_{L}(Z)=F-S(Z)(L)$.

Notice that $\iota^{*} E_{L}$ is a first integral, with corresponding vector field $\Gamma$, though the proposition is vacuous in this case; however, it is clear from the facts that $\Gamma\lrcorner \iota^{*} \omega_{L}+d\left(\iota^{*} E_{L}\right) \in \tilde{\mathcal{D}}^{\circ}$ and $\Gamma \in \tilde{\mathcal{D}}$ that $\Gamma\left(\iota^{*} E_{L}\right)=0$.

We can now consider the question of whether there is any correlation between conserved quantities and symmetries for constrained systems. By an infinitesimal symmetry of $\Gamma$ we mean a vector field $Z$ tangent to $\mathcal{C}$ such that $\mathcal{L}_{Z} \Gamma=0$. We shall give two different sets of conditions for deriving a symmetry from a first integral: the first result involves conditions on $\Gamma$, the second on the vector field $Z_{f} \in \tilde{\mathcal{D}}$ corresponding to a first integral $f$. We derive them both as corollaries of the following proposition.

Proposition 5. Let $Z$ be a vector field tangent to $\mathcal{C}$ such that $\tilde{\mathcal{D}}\lrcorner \mathcal{L}_{Z}\left(\iota^{*} \omega_{L}\right) \subset \tilde{\mathcal{D}}^{\circ}$ and $\mathcal{L}_{Z}(\tilde{\mathcal{D}}) \subset$ $\tilde{\mathcal{D}}$. Let $f$ be any function on $\mathcal{C}$ and $Z_{f} \in \tilde{\mathcal{D}}$ the corresponding vector field. Then $\left.\mathcal{L}_{Z}\left(Z_{f}\right)\right\lrcorner \iota^{*} \omega_{L}-$ $d(Z(f)) \in \tilde{\mathcal{D}}^{\circ}$. If, further, $Z(f)=0$ then $\mathcal{L}_{Z}\left(Z_{f}\right)=0$.

Proof. We have $\left.Z_{f}\right\lrcorner \iota^{*} \omega_{L}-d f=\phi \in \tilde{\mathcal{D}}^{\circ}$. Take the Lie derivative with respect to $Z$ to obtain

$$
\left.\left.\mathcal{L}_{Z}\left(Z_{f}\right)\right\lrcorner \iota^{*} \omega_{L}-d(Z(f))=-Z_{f}\right\lrcorner \mathcal{L}_{Z}\left(\iota^{*} \omega_{L}\right)+\mathcal{L}_{Z} \phi
$$

Since $Z_{f} \in \tilde{\mathcal{D}}$, by assumption the first term on the right-hand side belongs to $\tilde{\mathcal{D}}^{\circ}$. Now for any $Y \in \tilde{\mathcal{D}}$

$$
\mathcal{L}_{Z} \phi(Y)=Z(\phi(Y))-\phi\left(\mathcal{L}_{Z} Y\right)=0
$$

since $\mathcal{L}_{Z} Y \in \tilde{\mathcal{D}}$ by assumption. Thus $\mathcal{L}_{Z} \phi \in \tilde{\mathcal{D}}^{\circ}$. So $\left.\mathcal{L}_{Z}\left(Z_{f}\right)\right\lrcorner \iota^{*} \omega_{L}-d(Z(f)) \in \tilde{\mathcal{D}}^{\circ}$. If $Z(f)=0$ (or indeed if $Z(f)$ is constant) then $\mathcal{L}_{Z}\left(Z_{f}\right) \in \tilde{\mathcal{D}}$ while $\left.\mathcal{L}_{Z}\left(Z_{f}\right)\right\lrcorner \iota^{*} \omega_{L} \in \tilde{\mathcal{D}}^{\circ}$, and so $\mathcal{L}_{Z}\left(Z_{f}\right)=0$ by Corollary 1 .

In the course of the proof we have in effect established that $\mathcal{L}_{Z}(\tilde{\mathcal{D}}) \subset \tilde{\mathcal{D}}$ if and only if $\mathcal{L}_{Z}\left(\tilde{\mathcal{D}}^{\circ}\right) \subset$ $\tilde{\mathcal{D}}^{\circ}$.

The condition $\tilde{\mathcal{D}}\lrcorner \mathcal{L}_{Z}\left(\iota^{*} \omega_{L}\right) \subset \tilde{\mathcal{D}}^{\circ}$ is equivalent to $\mathcal{L}_{Z}\left(\iota^{*} \omega_{L}\right)(\tilde{\mathcal{D}}, \tilde{\mathcal{D}})=0$, or in other words the distribution $\tilde{\mathcal{D}}$ is isotropic for $\mathcal{L}_{Z}\left(\iota^{*} \omega_{L}\right)$; but in view of the appeal to Corollary 1 the formulation in the statement of the proposition seems preferable.

Corollary 2. Suppose that $\Gamma$ satisfies the conditions specified for $Z$ in the proposition above. Then for any first integral $f, Z_{f}$ is a symmetry of $\Gamma$.

Proof. Since $\Gamma(f)=0, \mathcal{L}_{Z_{f}} \Gamma=-\mathcal{L}_{\Gamma}\left(Z_{f}\right)=0$.

Corollary 3. Let $f$ be a first integral of $\Gamma, Z_{f}$ the corresponding vector field. Suppose that $Z_{f}$ satisfies the conditions specified for $Z$ in the proposition above. Then $Z_{f}$ is a symmetry of $\Gamma$. 
Proof. A certain amount of mental gymnastics is required here: we take $Z_{f}$ for $Z$ in the proposition above, and $\Gamma$ for $Z_{f}$ (that is, we take $-\iota^{*} E_{L}$ for $f$ ). Since $f$ is a first integral, $Z_{f}\left(\iota^{*} E_{L}\right)=0$, and the final conclusion of the proposition holds.

From the second of these corollaries we obtain the following theorem, which gives sufficient conditions on a vector field $Z \in \tilde{\mathcal{D}}$ for it to both be a symmetry and generate a first integral.

Theorem 2. Let $Z \in \tilde{\mathcal{D}}$ be such that $\tilde{\mathcal{D}}\lrcorner \mathcal{L}_{Z}\left(\iota^{*} \omega_{L}\right) \subset \tilde{\mathcal{D}}^{\circ}, \mathcal{L}_{Z}(\tilde{\mathcal{D}}) \subset \tilde{\mathcal{D}}, \mathcal{L}_{Z}\left(\iota^{*} \omega_{L}\right) \in d\left(\tilde{\mathcal{D}}^{\circ}\right)$, and $Z\left(\iota^{*} E_{L}\right)=0$. Then $Z$ is a symmetry of $\Gamma$, and there is, at least locally, a function $f$ on $\mathcal{C}$ such that $Z=Z_{f}$ and $\Gamma(f)=0$. The set of vector fields $Z$ satisfying these conditions forms a Lie algebra $\mathcal{S}$. For $Z_{1}, Z_{2} \in \mathcal{S}$, with corresponding first integrals $f_{1}, f_{2}$, we have $Z_{1}\left(f_{2}\right)=-Z_{2}\left(f_{1}\right)$, and the first integral corresponding to $\left[Z_{1}, Z_{2}\right]$ is (up to an additive constant) $Z_{1}\left(f_{2}\right)$.

Proof. The vector field $Z$ satisfies the conditions of Theorem 1 (see the remarks following it) and Corollary 3. If $Z_{1}, Z_{2} \in \mathcal{S}$, then $Z_{2} \in \tilde{\mathcal{D}}$ and $\mathcal{L}_{Z_{1}}(\tilde{\mathcal{D}}) \subset \tilde{\mathcal{D}}$, so $\left[Z_{1}, Z_{2}\right]=\mathcal{L}_{Z_{1}} Z_{2} \in \tilde{\mathcal{D}}$. It is easy to see that $\left[Z_{1}, Z_{2}\right]$ satisfies the other conditions, and also that $k_{1} Z_{1}+k_{2} Z_{2}, k_{1}, k_{2} \in \mathbb{R}$, satisfies the conditions. Thus $\mathcal{S}$ is a Lie algebra. We have $Z_{1}\left(f_{2}\right)=\iota^{*} \omega_{L}\left(Z_{1}, Z_{2}\right)=-Z_{2}\left(f_{1}\right)$. From Proposition 5 we see that $\left.\left[Z_{1}, Z_{2}\right]\right\lrcorner \iota^{*} \omega_{L}-d\left(Z_{1}\left(f_{2}\right)\right) \in \tilde{\mathcal{D}}^{\circ}$.

\subsection{Systems with maximally nonintegrable constraint distributions}

The statements in the previous sections can be further refined if one is in the situation where the distribution is 'as non-integrable as it can be' (in a sense we shall explain next). We shall show that one may actually assume without loss of generality that this is always the case.

For any distribution $\mathcal{D}$ on $Q$ let $[\mathcal{D}]$ be the smallest involutive distribution containing $\mathcal{D}$; it consists of linear combinations of repeated brackets of vector fields in $\mathcal{D}$, as the notation is designed to suggest. If $[\mathcal{D}]=\mathfrak{X}(Q)$ we say that $\mathcal{D}$ is maximally nonintegrable. Other authors use other adjectives, such as e.g. 'totally nonholonomic' [18] or 'completely nonholonomic' [31].

Let $\mathcal{D}^{\prime}$ be the distribution on $T Q$ spanned by all vector fields $X^{\mathrm{C}}$ and $X^{\mathrm{V}}$ for $X \in \mathcal{D}$. Then $\mathcal{D}^{\prime}$ has the properties that $\tau_{*} \mathcal{D}^{\prime}=\mathcal{D}$ and $S\left(\mathcal{D}^{\prime}\right)=\mathcal{V} \cap \mathcal{D}^{\prime}$ (where $\mathcal{V}$ is the vertical distribution), and it is determined by these properties. If $\mathcal{D}$ is involutive so is $\mathcal{D}^{\prime}$, since for any $X, Y \in \mathcal{D}$, $\left[X^{\mathrm{C}}, Y^{\mathrm{C}}\right]=[X, Y]^{\mathrm{C}} \in \mathcal{D}^{\prime},\left[X^{\mathrm{C}}, Y^{\mathrm{V}}\right]=[X, Y]^{\mathrm{V}} \in \mathcal{D}^{\prime}$, and of course $\left[X^{\mathrm{V}}, Y^{\mathrm{V}}\right]=0$. Note that $\mathfrak{X}(Q)^{\prime}=\mathfrak{X}(T Q)$.

\section{Proposition 6.}

$$
[\mathcal{D}]^{\prime}=\left[\mathcal{D}^{\prime}\right]
$$

Proof. Evidently $\mathcal{D}^{\prime} \subset[\mathcal{D}]^{\prime}$ and $[\mathcal{D}]^{\prime}$ is involutive, so $\left[\mathcal{D}^{\prime}\right] \subset[\mathcal{D}]^{\prime}$. On the other hand, $\left[\mathcal{D}^{\prime}\right]$ is spanned by the repeated brackets of complete and vertical lifts of vector fields in $\mathcal{D}$, and these (when nonzero) are complete or vertical lifts of vector fields in $[\mathcal{D}]:$ so $[\mathcal{D}]^{\prime} \subset\left[\mathcal{D}^{\prime}\right]$.

Corollary 4. If $\mathcal{D}$ is maximally nonintegrable so is $\mathcal{D}^{\prime}$.

Now consider, for a constrained system with constraint distribution $\mathcal{D}$, those conserved quantities which are just functions on $Q$. Of course in the unconstrained case there aren't any; but in the constrained case the condition for $f$ to be conserved is just that $X(f)=0$ for all $X \in \mathcal{D}$. Then evidently $X(f)=0$ for $X \in[\mathcal{D}]$ (see [20] for a similar statement). So these conserved quantities 
are constant on the integral submanifolds of $[\mathcal{D}]$; and conversely, since $\mathcal{D} \subset[\mathcal{D}]$. So the leaves (maximal connected integral submanifolds) of $[\mathcal{D}]$ are the level sets of (an independent subset of) the conserved quantities $f$.

Let us restrict everything to a leaf $\mathrm{E}$ of $[\mathcal{D}]$. Note that $\mathcal{D}$ is still a distribution on $\mathrm{E}$; and since the base integral curves of $\Gamma$ are everywhere tangent to $\mathcal{D}$, if they start in $€$ they lie in $\mathrm{E}$.

Proposition 7. Let $E$ be a leaf of $[\mathcal{D}], \bar{L}, \overline{\mathcal{D}}$ the restrictions of $L$ and $\mathcal{D}$ to $E$, then (assuming that $L$ is regular with respect to $\mathcal{D})$ the dynamical field of the constrained system on $E$ defined by $\bar{L}$ and $\overline{\mathcal{D}}$ is just the restriction of $\Gamma$ to $T E \cap \mathcal{C}$.

Proof. We can identify $T €$ with the leaf of $\left[\mathcal{D}^{\prime}\right]$ in $T Q$ which projects onto $\mathrm{E}$, and the constraint submanifold $\overline{\mathcal{C}} \subset T \mathrm{E}$ corresponding to $\overline{\mathcal{D}}$ with $T \mathrm{E} \cap \mathcal{C}$. We know that $\Gamma$ belongs to $\mathcal{D}^{\prime}$, and is therefore tangent to $\overline{\mathcal{C}}$. Its restriction to $\overline{\mathcal{C}}$ is uniquely determined by the restriction of the equations $\Gamma\left(X^{\mathrm{V}}(L)\right)-X^{\mathrm{C}}(L)=0, X \in \mathcal{D}$, to $\overline{\mathcal{C}}$; but these are just the Lagrange-d'Alembert equations for the system on $\mathrm{E}$.

So without essential loss of generality we may assume that $\mathcal{D}$ is maximally nonintegrable: if not we just have to restrict to a leaf $\mathrm{\epsilon}$ of $[\mathcal{D}]$. Suppose coordinates have been chosen on $Q$ so that the leaves of $[\mathcal{D}]$ are given by $x^{r}=$ constant for an appropriate range of values of $r$ : then these coordinates will of course appear in the expressions for base the integral curves of the restriction of $\Gamma$ to $\mathrm{E}$, but only as parameters which take the constant values appropriate to $\mathrm{E}$, the leaf in which the curve lies.

Let $\tilde{\mathcal{D}}$ be as in the previous section. We also need to identify $[\tilde{\mathcal{D}}]$, the smallest involutive distribution on $\mathcal{C}$ containing $\tilde{\mathcal{D}}$. Now for any projectable vector fields $Z_{1}, Z_{2}$ on $\mathcal{C},\left[Z_{1}, Z_{2}\right]$ is projectable, and $\tau_{\mid \mathcal{C} *}\left[Z_{1}, Z_{2}\right]=\left[\tau_{\mid \mathcal{C} *} Z_{1}, \tau_{\mid \mathcal{C} *} Z_{2}\right]$. Since $[\tilde{\mathcal{D}}]$ is spanned by repeated brackets of local basis vector fields of $\tilde{\mathcal{D}}$, it is projectable to $Q$, and its projection is an involutive distribution containing $\mathcal{D}$. But by construction $\tau_{\mid \mathcal{C} *}[\tilde{\mathcal{D}}]$ is spanned by repeated brackets of vector fields in $\mathcal{D}$, so $\tau_{\mid \mathcal{C} *}[\tilde{\mathcal{D}}] \subset[\mathcal{D}]$, whence $\tau_{\mid \mathcal{C} *}[\tilde{\mathcal{D}}]=[\mathcal{D}]$. Clearly the kernel of $[\tilde{\mathcal{D}}]$ under projection is $\mathcal{D}^{\mathrm{V}}$. So $[\tilde{\mathcal{D}}]$ is determined by the fact that $\tau_{\mid \mathcal{C} * *}[\tilde{\mathcal{D}}]=[\mathcal{D}]$; that is, $[\tilde{\mathcal{D}}]_{u}=\tau_{\mid \mathcal{C} * u}{ }^{-1}\left([\mathcal{D}]_{\tau(u)}\right)$. In particular, if $\mathcal{D}$ is maximally nonintegrable so is $\tilde{\mathcal{D}}$.

Clearly if $Z \in \tilde{\mathcal{D}}$ is such that $\mathcal{L}_{Z}\left(\iota^{*} \omega_{L}\right)=0$, so that $Z$ is a symmetry of $\iota^{*} \omega_{L}$, and $\mathcal{L}_{Z}(\tilde{\mathcal{D}}) \subset \tilde{\mathcal{D}}$ and $Z\left(\iota^{*} E_{L}\right)=0$, then $Z$ satisfies the conditions of Theorem 2, When the constraint distribution is maximally nonintegrable we have the following partial converses. In the first we assume that $\mathcal{D}$ is 2-step maximally nonintegrable. A distribution $\mathcal{D}$ on a manifold $Q$ is 2-step maximally nonintegrable if $\mathcal{D}+[\mathcal{D}, \mathcal{D}]=\mathfrak{X}(Q)$.

Proposition 8. If $\mathcal{D}$ is 2-step maximally nonintegrable then a vector field $Z \in \tilde{\mathcal{D}}$ satisfying $\tilde{\mathcal{D}}\lrcorner \mathcal{L}_{Z}\left(\iota^{*} \omega_{L}\right) \in \tilde{\mathcal{D}}^{\circ}, \mathcal{L}_{Z}(\tilde{\mathcal{D}}) \subset \tilde{\mathcal{D}}$ and $\mathcal{L}_{Z}\left(\iota^{*} \omega_{L}\right) \in d\left(\tilde{\mathcal{D}}^{\circ}\right)$ is a symmetry of $\iota^{*} \omega_{L}$.

Proof. We have $\mathcal{L}_{Z}\left(\iota^{*} \omega_{L}\right)=d \phi$ for some $\phi \in \tilde{\mathcal{D}}^{\circ}$ such that $\left.Y\right\lrcorner d \phi \in \tilde{\mathcal{D}}^{\circ}$ for all $Y \in \tilde{\mathcal{D}}$, or $d \phi\left(Y_{1}, Y_{2}\right)=0$ for all $Y_{1}, Y_{2} \in \tilde{\mathcal{D}}$. But for $Y_{1}, Y_{2} \in \tilde{\mathcal{D}}, d \phi\left(Y_{1}, Y_{2}\right)=-\phi\left(\left[Y_{1}, Y_{2}\right]\right)$. If $\mathcal{D}$ is 2-step maximally nonintegrable, so is $\tilde{\mathcal{D}}$. So $\phi$ vanishes on $\mathfrak{X}(\mathcal{C})$, that is, $\mathcal{L}_{Z}\left(\iota^{*} \omega_{L}\right)=0$.

For the second result we need to strengthen the first condition of the theorem.

Proposition 9. If $\mathcal{D}$ is maximally nonintegrable then a vector field $Z \in \tilde{\mathcal{D}}$ satisfying $\tilde{\mathcal{D}}\lrcorner \mathcal{L}_{Z}\left(\iota^{*} \omega_{L}\right)=$ $0, \mathcal{L}_{Z}(\tilde{\mathcal{D}}) \subset \tilde{\mathcal{D}}$ and $\mathcal{L}_{Z}\left(\iota^{*} \omega_{L}\right) \in d\left(\tilde{\mathcal{D}}^{\circ}\right)$ is a symmetry of $\iota^{*} \omega_{L}$. 
Proof. We have $\mathcal{L}_{Z}\left(\iota^{*} \omega_{L}\right)=d \phi$ for some $\phi \in \tilde{\mathcal{D}}^{\circ}$ such that $\left.Y\right\lrcorner d \phi=0$ for all $Y \in \tilde{\mathcal{D}}$. We therefore consider the set $\mathcal{S}$ of those 1-forms $\psi \in \tilde{\mathcal{D}}^{\circ}$ such that $\left.Y\right\lrcorner d \psi=0$ for all $Y \in \tilde{\mathcal{D}}$, or equivalently $\mathcal{L}_{Y} \psi=0$ for all $Y \in \tilde{\mathcal{D}}$. It is an $\mathbb{R}$-linear subspace of $\tilde{\mathcal{D}}^{\circ}$, and indeed a module over functions invariant under $\tilde{\mathcal{D}}$. Now if $Y$ is any vector field on $\mathcal{C}$ such that $\psi(Y)=0$ and $\mathcal{L}_{Y} \psi=0$, and $Y^{\prime}=f Y$ for any function $f$ on $\mathcal{C}$, then $\psi\left(Y^{\prime}\right)=0$, and

$$
\left.\left.\mathcal{L}_{Y^{\prime}} \psi=f(Y\lrcorner d \psi\right)+d(f Y\lrcorner \psi\right)=f \mathcal{L}_{Y} \psi=0 .
$$

Moreover, if $Y_{1}, Y_{2}$ satisfy $\psi\left(Y_{1}\right)=\psi\left(Y_{2}\right)=0$ and $\mathcal{L}_{Y_{1}} \psi=\mathcal{L}_{Y_{2}} \psi=0$ then

$$
\psi\left(\left[Y_{1}, Y_{2}\right]\right)=Y_{1}\left(\psi\left(Y_{2}\right)\right)-\mathcal{L}_{Y_{1}} \psi\left(Y_{2}\right)=0,
$$

and

$$
\mathcal{L}_{\left[Y_{1}, Y_{2}\right]} \psi=\left[\mathcal{L}_{Y_{1}}, \mathcal{L}_{Y_{2}}\right] \psi=0 .
$$

So if $[\tilde{\mathcal{D}}]$ is the smallest involutive distribution containing $\tilde{\mathcal{D}}$ then $\mathcal{S} \subset[\tilde{\mathcal{D}}]^{\circ}$. But if $\mathcal{D}$ is maximally nonintegrable then $[\tilde{\mathcal{D}}]=\mathfrak{X}(\mathcal{C})$ and so $\mathcal{S}=\{0\}$. Thus $\mathcal{L}_{Z}\left(\iota^{*} \omega_{L}\right)=0$.

\section{The nonholonomic Noether theorem}

The title of this section refers to the terminology used in the paper [21] by Fassò et al. We shall first re-express their version of the theorem in the current framework. Next we shall show how it relates to the Cartan form approach.

\subsection{The theorem and the reaction-annihilator distribution}

In 11 we defined (for any second-order field $\Gamma$ ) a 1-form $\varepsilon$ along the tangent bundle projection (restricted to $\mathcal{C}$ in the nonholonomic case) by $\varepsilon(X)=\Gamma\left(X^{\mathrm{V}}(L)\right)-X^{\mathrm{C}}(L)$ for $X$ a vector field on $Q$. The Lagrange-d'Alembert principle is that there is a unique $\Gamma$ of second-order type, tangent to $\mathcal{C}$, such that $\varepsilon$ annihilates $\mathcal{D}$. The form $\varepsilon$ corresponding to that particular $\Gamma$ is what Fassò et al., in [21], call the reaction set $\mathcal{R}$. The idea of Fassò et al. is that there may be vector fields $Z$ on $Q$, not necessarily in $\mathcal{D}$, such that $\varepsilon(Z)=0$; such a vector field belongs to the so-called reaction-annihilator distribution $\mathcal{R}^{\circ}$. The next statement is the nonholonomic Noether theorem of [19, 21, 22].

Theorem 3. For a vector field $Z$ on $Q$ any two of the following three conditions imply the third: (1) $Z^{\mathrm{C}}(L)=0$ on $\mathcal{C}$; (2) $\varepsilon(Z)=0$; (3) $\left.Z^{\mathrm{V}}(L)\right|_{\mathcal{C}}$ is a first integral of $\Gamma$.

Proof. The proof is straightforward: we have $\Gamma\left(Z^{\mathrm{V}}(L)\right)=Z^{\mathrm{C}}(L)+\varepsilon(Z)$, so if any two of the terms vanish so does the third.

We may equivalently express matters in terms of multipliers. Let $\left\{X_{\alpha}, X_{a}\right\}$ be a basis of vector fields on $Q$, where the $X_{\alpha}$ span $\mathcal{D}$. Evidently $\varepsilon\left(X_{\alpha}\right)=0$ on $\mathcal{C}$, while $\varepsilon\left(X_{a}\right)=\lambda_{a}$, for some functions $\lambda_{a}$ on $\mathcal{C}$. These $\lambda_{a}$ play the role of the Lagrangian multipliers one finds in many formulations of the equations of nonholonomic dynamics. Let $Z=Z^{a} X_{a}+Z^{\alpha} X_{\alpha}$; then $Z$ is in $\mathcal{R}^{\circ}$ if and only if $Z^{a} \lambda_{a}=0$. By definition, $Z^{a} \lambda_{a}=\Gamma\left(Z^{a} X_{a}^{\mathrm{V}}(L)\right)-\left(Z^{a} X_{a}\right)^{\mathrm{C}}(L)$. If now $Z^{\mathrm{C}}(L)=0$, then $\left(Z^{a} X_{a}\right)^{\mathrm{C}}(L)=-\left(Z^{\alpha} X_{\alpha}\right)^{\mathrm{C}}(L)$. Replacing this above, and taking into account 
the fact that $\Gamma$ is such that $\left(Z^{\alpha} X_{\alpha}\right)^{\mathrm{C}}(L)=\Gamma\left(Z^{\alpha} X_{\alpha}^{\mathrm{V}}(L)\right)$, we easily get that $Z^{a} \lambda_{a}=\Gamma\left(Z^{\mathrm{V}}(L)\right)$, and the result follows.

In this general situation, the conserved momentum $Z^{\mathrm{v}}(L)$ may depend on the component of $Z$ transverse to $\mathcal{D}$.

We could introduce the following small improvement to the above theorem: for any function $f$ on $Q$,

$$
\varepsilon(X)=\Gamma\left(X^{\mathrm{V}}(L)-f\right)-\left(X^{\mathrm{C}}(L)-\dot{f}\right)
$$

if any two of the three terms vanish so does the third. If $L$ is of mechanical type (meaning that it is of the form $T-V$, where $T$ is associated to a Riemannian metric, and $V$ is a potential) this adds nothing new, since if $X^{\mathrm{C}}(L)=\dot{f}$ then each side must be zero, by equating to zero the separate powers of $u$. Symmetries for which $X^{\mathrm{C}}(L)=\dot{f}$ are therefore only of interest for more general types of Lagrangians, e.g. for Lagrangians with magnetic terms. In that context, the Lagrangian is said to be quasi-invariant, see e.g. [30.

We shall now reinterpret the reaction-annihilator distribution $\mathcal{R}^{\circ}$. It is not immediately clear which manifold $\mathcal{R}^{\circ}$ is supposed to be a distribution on. For each point $(q, u)$ of $\mathcal{C}$, there is a subspace of $T_{q} Q$ consisting of vectors $v$ such that $\varepsilon_{(q, u)}(v)=0$ : but it will in general depend on $u$. For example, if the Lagrangian is of mechanical type with a potential term $\phi$, then $\lambda_{a}$ contains two terms, one quadratic in velocities and one independent of them: the first is what one gets from the kinetic energy term, the second is just $X_{a}(\phi)$ (see the computations in Section 5.2). So the set $\left\{v \in T_{q} Q: v^{a} \lambda_{a}(q, u)=0\right\}$ will depend on $u$, as we claimed above.

One may impose further conditions so that $\mathcal{R}^{\circ}$ can be regarded as a distribution on $Q$. Indeed, there may very well be vector fields $Z$ on $Q$ such that $Z^{a}(q) \lambda_{a}(q, u)=0$ for all $u \in \mathcal{C}_{q}$ (any $Z \in \mathcal{D}$ will do). In the case of a Lagrangian of mechanical type, since the $Z^{a}$ are functions of $q$ alone they must in fact satisfy two conditions: the one coming from the quadratic part of $\lambda_{a}$, and in addition $Z^{a} X_{a}(\phi)=0$. It will become clear immediately below that to restrict attention to vector fields on $Q$ in this way (i.e. to think of $\mathcal{R}^{\circ}$ as a distribution on $Q$ ) is to impose an unnecessary limitation, so we shall not insist on it.

We can reinterpret $\varepsilon$ in terms of the fibre metric, much as we did in Section 2, In fact for any vector field $X$ on $Q$,

$$
\varepsilon(X)=\Gamma\left(X^{\mathrm{V}}(L)\right)-X^{\mathrm{C}}(L)=\left(\Gamma-\Gamma_{0}\right)\left(X^{\mathrm{V}}(L)\right)=g\left(\Gamma-\Gamma_{0}, X^{\mathrm{V}}\right) .
$$

From this perspective it is clear that if we require $\mathcal{R}^{\circ}$ to be in some sense a distribution, it must be interpreted as the distribution of vector fields along $\iota$ which are fibre-normal to $\Gamma-\Gamma_{0}$. It will then consist of vertical vector fields on $\mathcal{C} \subset T Q$, rather than vector fields on $Q$; but it may contain vertical lifts of vector fields on $Q$, and it is these which are of interest from the point of view of Theorem 3 ,

\subsection{Relation to the Cartan form approach}

We now discuss the nonholonomic Noether theorem of Fassò et al. from the Cartan form point of view.

We first make one further interpretation of $\varepsilon$. Notice that $\Gamma\lrcorner \omega_{L}+d E_{L}$ is a semi-basic 1-form along $\mathcal{C}$, say $\epsilon$. For any $v \in T_{u}(T Q)$ (where $\left.u \in \mathcal{C}\right), \epsilon_{u}(v)=g_{a b}(u) \vartheta^{a}(v) \gamma^{b}=g_{u}\left(S(v), \Gamma-\Gamma_{0}\right)$, since $S(v)=\vartheta^{\alpha}(v) \mathcal{Y}_{\alpha}+\vartheta^{a}(v) \mathcal{Y}_{a}$. That is to say, $\epsilon_{u}(v)=\varepsilon_{u}\left(\tau_{*} v\right)$, or in other words $\epsilon$ is $\varepsilon$ 
considered as a semi-basic 1 -form along $\mathcal{C}$. The element of $\tilde{\mathcal{D}}^{\circ}$ determined by $\left.\Gamma\right\lrcorner \iota^{*} \omega_{L}+d\left(\iota^{*} E_{L}\right)$ according to Proposition 4 is $\iota^{*} \epsilon$.

Recall that for any vector field $X$ on $Q$, the vector field $X^{\mathrm{C}}$ satisfies $\mathcal{L}_{X^{\mathrm{C}}} S=0$ and $\left[\Delta, X^{\mathrm{C}}\right]=0$, where $\Delta$ is the Liouville field.

For any Lagrangian system (with Cartan forms $\theta_{L}$ and $\omega_{L}=d \theta_{L}$ )

$$
\begin{aligned}
\left.X^{\mathrm{C}}\right\lrcorner \omega_{L} & \left.=X^{\mathrm{C}}\right\lrcorner d \theta_{L} \\
& \left.=\mathcal{L}_{X^{\mathrm{C}}} \theta_{L}-d\left(X^{\mathrm{C}}\right\lrcorner \theta_{L}\right) \\
& =\mathcal{L}_{X^{\mathrm{C}}}(S(d L))-d\left(X^{\mathrm{V}}(L)\right) \\
& =S\left(d\left(X^{\mathrm{C}}(L)\right)\right)-d\left(X^{\mathrm{V}}(L)\right) .
\end{aligned}
$$

This holds everywhere on $T Q$, and regardless of whether $L$ is regular.

Secondly,

$$
\begin{aligned}
\varepsilon(X)=\epsilon\left(X^{\mathrm{C}}\right) & \left.=(\Gamma\lrcorner \omega_{L}+d E_{L}\right)\left(X^{\mathrm{C}}\right) \\
& \left.=-\left(X^{\mathrm{C}}\right\lrcorner \omega_{L}\right)(\Gamma)+X^{\mathrm{C}}\left(E_{L}\right) \\
& =-\Delta\left(X^{\mathrm{C}}(L)\right)+\Gamma\left(X^{\mathrm{V}}(L)\right)+X^{\mathrm{C}}(\Delta(L))-X^{\mathrm{C}}(L) \\
& =\Gamma\left(X^{\mathrm{V}}(L)\right)-X^{\mathrm{C}}(L)
\end{aligned}
$$

as expected. This holds along $\mathcal{C}$, but neither $X^{\mathrm{C}}$ nor $X^{\mathrm{V}}$ need be tangent to $\mathcal{C}$.

Thirdly, we propose an analogue of Theorem 3 ,

Theorem 4. For any vector field $Z$ tangent to $\mathcal{C}$ and for any function $f$ on $\mathcal{C}$ such that $Z\lrcorner \iota^{*} \omega_{L}-$ df $\in \tilde{\mathcal{D}}^{\circ}$, we have

$$
\Gamma(f)=Z\left(\iota^{*} E_{L}\right)-\iota^{*} \epsilon(Z)
$$

and if any two of the terms vanish so does the third.

Proof. This is a small generalization of the proof of Theorem 1

(Note in passing that since $\left.\iota^{*} \epsilon\left(Z_{f}\right)=(\Gamma\lrcorner \iota^{*} \omega_{L}+d\left(\iota^{*} E_{L}\right)\right)\left(Z_{f}\right)$ and $\left.\Gamma\right\lrcorner \iota^{*} \omega_{L}+d\left(\iota^{*} E_{L}\right) \in \tilde{\mathcal{D}}^{\circ}$, $\iota^{*} \epsilon\left(Z_{f}\right)=0$. Moreover, if $\left.Z\right\lrcorner \iota^{*} \omega_{L} \in \tilde{\mathcal{D}}^{\circ}$ then evidently $\iota^{*} \epsilon(Z)=Z\left(\iota^{*} E_{L}\right)$.)

We shall rederive Theorem 3 from the displayed formula in the statement of Theorem 4, that is, we shall show that Theorem 3 is a special case of our analogue theorem. Naively, one would like to substitute $X^{\mathrm{C}}$ for $Z$ : but this is not permissible since $X^{\mathrm{C}}$ is not necessarily tangent to $\mathcal{C}$, and in any case $X^{\mathrm{C}}$ does not correspond directly to $X^{\mathrm{V}}(L)$ via $\omega_{L}$. Let us denote by $\bar{X}^{\mathrm{C}}$ the projection of $X^{\mathrm{C}}$ onto $\mathcal{C}$ along the $\mathcal{Y}_{a}$, and let us set

$$
Z=-\bar{X}^{\mathrm{C}}+g^{\alpha \beta} \mathcal{Y}_{\beta}\left(X^{\mathrm{C}}(L)\right) \mathcal{Y}_{\alpha}
$$

Then $Z$ is tangent to $\mathcal{C}$ and satisfies $Z\lrcorner \iota^{*} \omega_{L}-d\left(\iota^{*}\left(X^{\mathrm{v}}(L)\right)\right) \in \tilde{\mathcal{D}}^{\circ}$. To see the latter, note that $S\left(d\left(X^{\mathrm{C}}(L)\right)\right)$ is semi-basic and $S\left(d\left(X^{\mathrm{C}}(L)\right)\right)\left(\mathcal{X}_{\alpha}\right)=\mathcal{Y}_{\alpha}\left(X^{\mathrm{C}}(L)\right)$, whence $S\left(d\left(X^{\mathrm{C}}(L)\right)\right)$ $\mathcal{Y}_{\alpha}\left(X^{\mathrm{C}}(L)\right) \vartheta^{\alpha} \in\left\langle\vartheta^{a}\right\rangle$. Recall the formula

$$
\omega_{L}=g_{\alpha \beta} \varphi^{\alpha} \wedge \vartheta^{\beta}+g_{a b} \varphi^{a} \wedge \vartheta^{b}+\frac{1}{2} \omega_{a b} \vartheta^{a} \wedge \vartheta^{b} .
$$


We have, on $\mathcal{C}$,

$$
\begin{aligned}
Z\lrcorner \omega_{L} & \left.=-X^{\mathrm{C}}\right\lrcorner \omega_{L}+\mathcal{Y}_{\alpha}\left(X^{\mathrm{C}}(L)\right) \vartheta^{\alpha} \quad\left(\bmod \vartheta^{a}\right) \\
& =-S\left(d\left(X^{\mathrm{C}}(L)\right)\right)+d\left(X^{\mathrm{V}}(L)\right)+\mathcal{Y}_{\alpha}\left(X^{\mathrm{C}}(L)\right) \vartheta^{\alpha} \quad\left(\bmod \vartheta^{a}\right) \\
& =d\left(X^{\mathrm{V}}(L)\right) \quad\left(\bmod \vartheta^{a}\right),
\end{aligned}
$$

whence $Z\lrcorner \iota^{*} \omega_{L}-d\left(\iota^{*}\left(X^{\mathrm{V}}(L)\right)\right) \in \tilde{\mathcal{D}}^{\circ}$. Now since $\epsilon$ is semi-basic, $\iota^{*} \epsilon(Z)=-\epsilon\left(X^{\mathrm{C}}\right)=-\varepsilon(X)$. It remains to calculate $Z\left(\iota^{*} E_{L}\right)$. For this purpose we require the following general result. Let $V$ be any vertical vector field. Then from the general formula for the fibre metric $g$

$$
g(V, \Delta)=V(\Delta(L))-\nabla_{V}^{0} \Delta(L)=V(\Delta(L))-V(L)=V\left(E_{L}\right),
$$

since $\nabla_{V}^{0} \Delta=V$. Thus on $\mathcal{C}$

$$
\begin{aligned}
Z\left(E_{L}\right) & =\left(-\bar{X}^{\mathrm{C}}+g^{\alpha \beta} \mathcal{Y}_{\beta}\left(X^{\mathrm{C}}(L)\right) \mathcal{Y}_{\alpha}\right)\left(E_{L}\right) \\
& =\left(-X^{\mathrm{C}}+\varphi^{a}\left(X^{\mathrm{C}}\right) \mathcal{Y}_{a}+g^{\alpha \beta} \mathcal{Y}_{\beta}\left(X^{\mathrm{C}}(L)\right) \mathcal{Y}_{\alpha}\right)\left(E_{L}\right) \\
& =-X^{\mathrm{C}}\left(E_{L}\right)+\varphi^{a}\left(X^{\mathrm{C}}\right) g\left(\mathcal{Y}_{a}, \Delta\right)+g^{\alpha \beta} \mathcal{Y}_{\beta}\left(X^{\mathrm{C}}(L)\right) g\left(\mathcal{Y}_{\alpha}, \Delta\right)
\end{aligned}
$$

Since the constraints are linear, $\Delta$ is tangent to $\mathcal{C}$, so $g\left(\mathcal{Y}_{a}, \Delta\right)=0$. If we write $\Delta=\nu^{\alpha} \mathcal{Y}_{\alpha}$ then $g\left(\mathcal{Y}_{\alpha}, \Delta\right)=g_{\alpha \beta} \nu^{\beta}$, and

$$
\begin{aligned}
g^{\alpha \beta} \mathcal{Y}_{\beta}\left(X^{\mathrm{C}}(L)\right) g\left(\mathcal{Y}_{\alpha}, \Delta\right) & =g^{\alpha \beta} \mathcal{Y}_{\beta}\left(X^{\mathrm{C}}(L)\right) g_{\alpha \gamma} \nu^{\gamma} \\
& =\nu^{\beta} \mathcal{Y}_{\beta}\left(X^{\mathrm{C}}(L)\right)=\Delta\left(X^{\mathrm{C}}(L)\right)
\end{aligned}
$$

It follows that on $\mathcal{C}, Z\left(E_{L}\right)=-X^{\mathrm{C}}\left(E_{L}\right)+\Delta\left(X^{\mathrm{C}}(L)\right)=X^{\mathrm{C}}(L)$, and therefore $Z\left(\iota^{*} E_{L}\right)=$ $\iota^{*}\left(X^{\mathrm{C}}(L)\right)$. So the formula $\Gamma(f)=Z\left(\iota^{*} E_{L}\right)-\iota^{*} \epsilon(Z)$ becomes $\Gamma\left(\iota^{*}\left(X^{\mathrm{V}}(L)\right)\right)=\iota^{*} X^{\mathrm{C}}(L)+\varepsilon(X)$. This is for a particular choice of $Z$ such that $Z\lrcorner \iota^{*} \omega_{L}-d\left(\iota^{*}\left(X^{\mathrm{V}}(L)\right)\right) \in \tilde{\mathcal{D}}^{\circ}$. For any other choice, say $Z^{\prime}$, we have $\left.\left(Z-Z^{\prime}\right)\right\lrcorner \iota^{*} \omega_{L} \in \tilde{\mathcal{D}}^{\circ}$, so that $Z^{\prime}\left(\iota^{*} E_{L}\right)-\iota^{*} \epsilon\left(Z^{\prime}\right)=Z\left(\iota^{*} E_{L}\right)-\iota^{*} \epsilon(Z)$, and the same conclusion holds.

The drawback of the approach in Theorem 4, however, is that the correspondence between first integrals and vector fields is no longer 1-1, as it was in Theorem 1.

\subsection{Special cases}

A particular question of interest is whether, and under what conditions, a complete lift $X^{\mathrm{C}}$ can satisfy the hypotheses of Theorem 2 on symmetries and first integrals. For this we require that

1. $X^{\mathrm{C}} \in \tilde{\mathcal{D}}$,

2. $\tilde{\mathcal{D}}\lrcorner \mathcal{L}_{X^{\mathrm{C}}}\left(\iota^{*} \omega_{L}\right) \subset \tilde{\mathcal{D}}^{\circ}$,

3. $\mathcal{L}_{X^{\mathrm{C}}}(\tilde{\mathcal{D}}) \subset \tilde{\mathcal{D}}$,

4. $\mathcal{L}_{X^{\mathrm{C}}}\left(\iota^{*} \omega_{L}\right) \in d\left(\tilde{\mathcal{D}}^{\circ}\right)$,

5. $X^{\mathrm{C}}\left(\iota^{*} E_{L}\right)=0$.

A couple of points of notation. 
- For a distribution $\mathcal{D}$, we denote by $\mathcal{D}^{1}$ its first derived distribution, which is the distribution spanned by $\mathcal{D}$ and brackets of vector fields in $\mathcal{D}$, that is, $\mathcal{D}^{1}=\mathcal{D}+[\mathcal{D}, \mathcal{D}]$. (Thus $\mathcal{D}$ is 2-step maximally nonintegrable just when $\mathcal{D}^{1}=\mathfrak{X}(Q)$.)

- We denote projectable vector fields on $T Q$, and more particularly on $\mathcal{C}$, with overbars; thus $\bar{Y}$ is projectable, and we set $\tau_{*} \bar{Y}=Y$. Note that $S(\bar{Y})=Y^{\mathrm{V}}$.

Lemma 1. A vector field $X$ on $Q$ is an infinitesimal symmetry of the distribution $\mathcal{D}$ (that is, it satisfies $\mathcal{L}_{X}(\mathcal{D}) \subset \mathcal{D}$ ) if and only if $X^{\mathrm{C}}$ is tangent to $\mathcal{C}$.

Proof. Let $\left(v^{a}, v^{\alpha}\right)$ be the quasi-velocities corresponding to the frame $\left\{X_{\alpha}, X_{a}\right\}$, as in [11. Then $v^{a}=0$ on $\mathcal{C}$. One easily verifies that $X^{\mathrm{C}}\left(v^{a}\right)=0$ if and only if $\left[X, X_{\alpha}\right]$ is of the form $A_{\alpha}^{\beta} X_{\beta}$.

Lemma 2. For a vector field $X$ on $Q$, if $X^{\mathrm{C}} \in \tilde{\mathcal{D}}$ then $\mathcal{L}_{X^{\mathrm{C}}}(\tilde{\mathcal{D}}) \subset \tilde{\mathcal{D}}$.

Proof. First of all, since $X^{\mathrm{C}}$ is evidently tangent to $\mathcal{C}, \mathcal{L}_{X}(\mathcal{D}) \subset \mathcal{D}$. We have to show that for any vector field $Z \in \tilde{\mathcal{D}}$, and for any $u \in \mathcal{C},\left(\mathcal{L}_{X^{\mathrm{C}}} Z\right)_{u}$ is tangent to $\mathcal{C}$ and $\tau_{*}\left(\mathcal{L}_{X^{\mathrm{C}}} Z\right)_{u} \in \mathcal{D}_{\tau(u)}$. It will be enough to consider those vector fields in $\tilde{\mathcal{D}}$ which are projectable. Let $\bar{Y}$ be any projectable vector field in $\tilde{\mathcal{D}}$, so that $Y \in \mathcal{D}$ : then $\mathcal{L}_{X^{\mathrm{C}}} \bar{Y}$ is tangent to $\mathcal{C}$ since both $X^{\mathrm{C}}$ and $\bar{Y}$ are; it is projectable, and its projection $\mathcal{L}_{X} Y$ belongs to $\mathcal{D}$.

So in this case condition 3 is superfluous.

Proposition 10. For a vector field $X$ on $Q, X^{\mathrm{C}}$ satisfies the conditions

1. $X^{\mathrm{C}} \in \tilde{\mathcal{D}}$,

2. $\tilde{\mathcal{D}}\lrcorner \mathcal{L}_{X^{\mathrm{C}}}\left(\iota^{*} \omega_{L}\right) \subset \tilde{\mathcal{D}}^{\circ}$,

3. $\mathcal{L}_{X^{\mathrm{C}}}\left(\iota^{*} \omega_{L}\right) \in d\left(\tilde{\mathcal{D}}^{\circ}\right)$,

4. $X^{\mathrm{C}}\left(\iota^{*} E_{L}\right)=0$,

if and only if

- $\mathcal{L}_{X}(\mathcal{D}) \subset \mathcal{D}$,

- $X \in \mathcal{D}$,

- there is a (locally defined) function $F$ on $Q$ such that $X^{\mathrm{C}}(L)=\dot{F}$ on $\mathcal{C}$,

- for any $Y \in \mathcal{D}^{1}, Y^{\mathrm{V}}\left(X^{\mathrm{C}}(L)\right)=Y(F)$ on $\mathcal{C}$.

(Notice that if $Y \in \mathcal{D}$, so that $Y^{\mathrm{V}}$ is tangent to $\mathcal{C}, Y^{\mathrm{V}}\left(X^{\mathrm{C}}(L)\right)=Y^{\mathrm{v}}(\dot{F})=Y(F)$ on $\mathcal{C}$, so the final condition is automatically satisfied. So that condition is really concerned with derivatives of $X^{\mathrm{C}}(L)$ in directions transverse to $\mathcal{C}$; that is, it says something about how $X^{\mathrm{C}}(L)$ changes as one moves off $\mathcal{C}$. Moreover, the transverse directions involved are those that arise from bracketing vector fields in $\mathcal{D}$.) 
Proof. As a preliminary step we evaluate $\mathcal{L}_{X^{\mathrm{C}}}\left(\iota^{*} \theta_{L}\right)$. Firstly, $\mathcal{L}_{X^{\mathrm{C}}} \theta_{L}=S\left(d\left(X^{\mathrm{C}}(L)\right)\right)$, whence $\mathcal{L}_{X^{\mathrm{C}}} \theta_{L}(\bar{Y})=Y^{\mathrm{V}}\left(X^{\mathrm{C}}(L)\right)$ and $\mathcal{L}_{X^{\mathrm{C}}} \theta_{L}$ vanishes on vertical vector fields (both of these assertions holding everywhere on $T Q)$. Thus $\mathcal{L}_{X^{\mathrm{C}}}\left(\iota^{*} \theta_{L}\right)$ vanishes on any vertical vector field tangent to $\mathcal{C}$, while for any $\bar{Y}$ tangent to $\mathcal{C}, \mathcal{L}_{X^{\mathrm{C}}}\left(\iota^{*} \theta_{L}\right)(\bar{Y})=\iota^{*}\left(Y^{\mathrm{V}}\left(X^{\mathrm{C}}(L)\right)\right)$.

Now suppose that $\mathcal{L}_{X}(\mathcal{D}) \subset \mathcal{D}, X \in \mathcal{D}$, and on $\mathcal{C}, X^{\mathrm{C}}(L)=\dot{F}$ and $Y^{\mathrm{V}}\left(X^{\mathrm{C}}(L)\right)=Y(F)$ for any $Y \in \mathcal{D}^{1}$. We show that the numbered conditions are satisfied.

Since $\mathcal{L}_{X}(\mathcal{D}) \subset \mathcal{D}, X^{\mathrm{C}}$ is tangent to $\mathcal{C}$. Furthermore, $\left.\tau\right|_{\mathcal{C}_{*}} X^{\mathrm{C}}=X \in \mathcal{D}$, so $X^{\mathrm{C}} \in \tilde{\mathcal{D}}$, which establishes that condition 1 is satisfied. For $\bar{Y} \in \tilde{\mathcal{D}}$, so that $Y^{\mathrm{v}}$ is tangent to $\mathcal{C}$, we have

$$
\mathcal{L}_{X^{\mathrm{C}}}\left(\iota^{*} \theta_{L}\right)(\bar{Y})=Y^{\mathrm{V}}\left(\iota^{*}\left(X^{\mathrm{C}}(L)\right)\right)=Y^{\mathrm{V}}(\dot{F})=Y(F)
$$

(strictly speaking, $\left.\tau\right|_{\mathcal{C}} ^{*}(Y(F))$ ). It follows that $\mathcal{L}_{X^{\mathrm{C}}}\left(\iota^{*} \theta_{L}\right)-d F \in \tilde{\mathcal{D}}^{\circ}$ (since both terms vanish on vertical vector fields). Thus $\mathcal{L}_{X^{\mathrm{C}}}\left(\iota^{*} \omega_{L}\right) \in d\left(\tilde{\mathcal{D}}^{\circ}\right)$, and condition 3 is satisfied. We next consider condition 2. It can be written $d \mathcal{L}_{X^{\mathrm{C}}}\left(\iota^{*} \theta_{L}\right)(\tilde{\mathcal{D}}, \tilde{\mathcal{D}})=0$. Using the usual formula for the exterior derivative it is easy to see that if either or both of the arguments is vertical then one gets zero. For $\bar{Y}_{1}, \bar{Y}_{2} \in \tilde{\mathcal{D}}$,

$$
\begin{aligned}
d \mathcal{L}_{X^{\mathrm{C}}}\left(\iota^{*} \theta_{L}\right)\left(\bar{Y}_{1}, \bar{Y}_{2}\right)= & \bar{Y}_{1}\left(\mathcal{L}_{X^{\mathrm{C}}}\left(\iota^{*} \theta_{L}\right)\left(\bar{Y}_{2}\right)-\bar{Y}_{2}\left(\mathcal{L}_{X^{\mathrm{C}}}\left(\iota^{*} \theta_{L}\right)\left(\bar{Y}_{1}\right)\right.\right. \\
& \quad-\mathcal{L}_{X^{\mathrm{C}}}\left(\iota^{*} \theta_{L}\right)\left(\left[\bar{Y}_{1}, \bar{Y}_{2}\right]\right) \\
= & \bar{Y}_{1}\left(Y_{2}(F)\right)-\bar{Y}_{2}\left(Y_{1}(F)\right)-\mathcal{L}_{X^{\mathrm{C}}}\left(\iota^{*} \theta_{L}\right)\left(\left[\bar{Y}_{1}, \bar{Y}_{2}\right]\right) \\
= & {\left[Y_{1}, Y_{2}\right](F)-\iota^{*}\left(\left[Y_{1}, Y_{2}\right]^{\mathrm{V}}\left(X^{\mathrm{C}}(L)\right)\right) } \\
= & 0
\end{aligned}
$$

because $Y^{\mathrm{V}}\left(X^{\mathrm{C}}(L)\right)=Y(F)$ on $\mathcal{C}$ for any $Y \in \mathcal{D}^{1}$. Finally, condition 4 follows directly from the fact that $\Delta(\dot{F})=\dot{F}$.

For the converse we shall make use of a frame $\left\{X_{i}\right\}$ on $Q$ with $\left\{X_{\alpha}\right\}$ a local basis for $\mathcal{D}$, as usual. We take vector fields $\bar{X}_{i}$ on (and tangent to) $\mathcal{C}$ projecting onto the $X_{i}$ : they could be the fibre-orthogonal projections onto $\mathcal{C}$ of the $X_{i}^{\mathrm{C}}$, for example. (These are not to be confused with the $\mathcal{X}_{\alpha}$ etc., which are not necessarily projectable.) Then $S\left(\bar{X}_{\alpha}\right)=X_{\alpha}^{\mathrm{v}}$, and $\left\{\bar{X}_{\alpha}, X_{\alpha}^{\mathrm{v}}\right\}$ is a basis for $\tilde{\mathcal{D}}$. We denote by $v^{i}$ the corresponding quasi-coodinates; $v^{a}=0$ on $\mathcal{C}$.

Suppose that $X^{\mathrm{C}}$ satisfies the numbered conditions. It follows from condition 1 , firstly that $X^{\mathrm{C}}$ is tangent to $\mathcal{C}$ and so $\mathcal{L}_{X}(\mathcal{D}) \subset \mathcal{D}$, and secondly that $X=\tau_{\mid \mathcal{C} *} X^{\mathrm{C}} \in \mathcal{D}$. Condition 3 implies the existence (locally) of a function $F$ on $\mathcal{C}$ such that $\mathcal{L}_{X^{\mathrm{C}}}\left(\iota^{*} \theta_{L}\right)-d F \in \tilde{\mathcal{D}}^{\circ}$. Since $\mathcal{L}_{X^{\mathrm{C}}}\left(\iota^{*} \theta_{L}\right)$ vanishes on any vertical vector field $V$ tangent to $\mathcal{C}$, and all such vector fields belong to $\tilde{\mathcal{D}}$, it follows that $V(F)=0$ for all such $V$, so $F$ is (the pull-back of) a function on $Q$. Then

$$
X_{\alpha}(F)=\mathcal{L}_{X^{\mathrm{C}}}\left(\iota^{*} \theta_{L}\right)\left(\bar{X}_{\alpha}\right)=X_{\alpha}^{\mathrm{V}}\left(X^{\mathrm{C}}(L)\right),
$$

whence by condition 4

$$
X^{\mathrm{C}}(L)=\Delta\left(X^{\mathrm{C}}(L)\right)=v^{\alpha} X_{\alpha}^{\mathrm{V}}\left(X^{\mathrm{C}}(L)\right)=v^{\alpha} X_{\alpha}(F)=\dot{F}
$$

on $\mathcal{C}$. From the calculation of $d \mathcal{L}_{X^{\mathrm{C}}}\left(\iota^{*} \theta_{L}\right)\left(\bar{Y}_{1}, \bar{Y}_{2}\right)$ above we see that on $\mathcal{C}$, for any $Y \in \mathcal{D}^{1}$, $Y^{\mathrm{V}}\left(X^{\mathrm{C}}(L)\right)=Y(F)$.

Corollary 5. The numbered conditions are satisfied if $X$ is a horizontal quasi-symmetry of the system, that is, if $\mathcal{L}_{X}(\mathcal{D}) \subset \mathcal{D}, X \in \mathcal{D}$, and for some function $F$ on $Q, X^{\mathrm{C}}(L)=\dot{F}$ holds on $T Q$. In fact $\mathcal{L}_{X^{\mathrm{C}}}\left(\iota^{*} \omega_{L}\right)=0$ in this case. 
Proof. We have $Y^{\mathrm{V}}\left(X^{\mathrm{C}}(L)\right)=Y(F)$ for any vector field $Y$ on $Q$. It follows that $\mathcal{L}_{X^{\mathrm{C}}}\left(\iota^{*} \theta_{L}\right)=$ $d F$.

We may conclude, from Theorem 2, that if $X$ is a horizontal quasi-symmetry then $X^{\mathrm{C}}$ is a symmetry of $\iota^{*} \omega_{L}$ and of $\Gamma$, and there is, at least locally, a function $f$ on $\mathcal{C}$ such that $X^{\mathrm{C}}=Z_{f}$ and $\Gamma(f)=0$. This result, with $f=F-\iota^{*}\left(X^{\mathrm{V}}(L)\right)$ of course, is well-known; the point of the exercise was to see how it is related to the theorem. To turn things around, we may say that the vector fields $Z$ satisfying the hypotheses of Theorem 2 should be regarded as generalizations of horizontal quasi-symmetries.

\section{Applications}

The bulk of the literature concentrates on the case where the Lagrangian is of mechanical type. So we now explain how the theory works in that special case. A simple subcase, but one which contains several instructive pointers to what happens in general, is furnished by submanifolds in Riemannian geometry.

\subsection{Conservation laws in Riemannian geometry}

First, some elementary remarks about geodesic conservation laws in Riemannian geometry.

Let $(M, g)$ be a Riemannian manifold, with Levi-Civita connection $\nabla$. For any vector field $Z$ on $M$, define a type $(0,2)$ tensor $K_{Z}$ by $K_{Z}(u, v)=g\left(\nabla_{u} Z, v\right)$. The necessary and sufficient condition for $Z$ to be a Killing field (infinitesimal isometry) is that $K_{Z}$ is skew-symmetric; in fact $\mathcal{L}_{Z} g(u, v)=K_{Z}(u, v)+K_{Z}(v, u)$.

For any curve $c$ in $M$, and any vector field $Z$,

$$
\frac{d}{d t}(g(Z, \dot{c}))=g\left(\nabla_{\dot{c}} Z, \dot{c}\right)+g\left(Z, \nabla_{\dot{c}} \dot{c}\right)=K_{Z}(\dot{c}, \dot{c})+g\left(Z, \nabla_{\dot{c}} \dot{c}\right) .
$$

If $c$ is an affinely parametrized geodesic, so that $\nabla_{\dot{c}} \dot{c}=0$, and $K_{Z}(\dot{c}, \dot{c})=0$, then $g(Z, \dot{c})$ is constant along $c$. If $Z$ is a Killing field then $g(Z, \dot{c})$ is constant along every geodesic; and conversely (since there is a geodesic in every direction, so $\dot{c}$ is an arbitrary vector). This, in fact, is Noether's theorem in Riemannian geometry: there is a 1-1 correspondence between geodesic invariants of the form $g(Z, \dot{c})$ and infinitesimal symmetries, that is, isometries or Killing fields.

Now consider the case of a Riemannian submanifold $N$ of a Riemannian manifold $(M, g)$ (the metric on $N$ is the restriction of $g$ ). The second fundamental form $\Pi$ is a type $(0,2)$ tensor on $N$ with values in the normal bundle, defined as follows. For any vector fields $\eta, \zeta$ on $N$, set $\Pi(\eta, \zeta)=\nabla_{\eta} \zeta^{\perp}$ (the normal component of $\nabla_{\eta} \zeta$ ). For any function $f$ on $N$ we have $\nabla_{\eta} f \zeta=$ $f \nabla_{\eta} \zeta+\eta(f) \zeta$, and so $\Pi(\eta, f \zeta)=f \Pi(\eta, \zeta)$, and $\Pi$ is tensorial. Moreover, $\nabla_{\eta} \zeta-\nabla_{\zeta} \eta=[\eta, \zeta]$ and the latter is tangent to $N$, which implies that $\Pi$ is symmetric in its arguments.

A curve $c$ on $N$ is geodesic with respect to the induced metric if and only if $\nabla_{\dot{c}} \dot{c}$ is normal to $N$ (here $\nabla$ is the Levi-Civita connection of $(M, g)$ ). If $c$ is geodesic then $\nabla_{\dot{c}} \dot{c}=\Pi(\dot{c}, \dot{c})$.

Now consider a vector field $Z$ defined in a neighbourhood of $N$ in $M$. Then for a geodesic $c$ in $N$

$$
\frac{d}{d t}(g(Z, \dot{c}))=K_{Z}(\dot{c}, \dot{c})+g\left(Z, \nabla_{\dot{c}} \dot{c}\right)=K_{Z}(\dot{c}, \dot{c})+g(Z, \Pi(\dot{c}, \dot{c})) .
$$


Of course if $Z$ is tangent to $N$ the last term vanishes and the situation reduces to the one discussed earlier. But suppose that $Z$ is not tangent to $N$, but satisfies the following two conditions: the restriction of $K_{Z}$ to $T N$ is skew, and $Z$ is orthogonal to the second fundamental form of $N$. (Of course the codimension of $N$ must be greater than 1 for this to be possible for $Z$ not tangent to $N$.) Then $g(Z, \dot{c})$ is constant along every geodesic of $N$. In fact any two of the following conditions implies the third:

1. $Z$ is orthogonal to the second fundamental form of $N$;

2. the restriction of $K_{Z}$ to $T N$ is skew;

3. $g(Z, \dot{c})$ is constant along every geodesic of $N$.

The first and last of these depend only on the values of $Z$ on $N$. Recall that $K_{Z}(u, v)=$ $g\left(\nabla_{u} Z, v\right)$, so that $K_{Z}$ involves derivatives of $Z$ : but since we are interested only in the case where $u$ and $v$ are tangent to $N$, we have to take derivatives only in directions tangent to $N$, so that $K_{Z}(u, v)$ also depends only on the values of $Z$ on $N$. In other words, one could state the result as follows: let $Z$ be a vector field on $N$ (but not necessarily tangent to it - that is, strictly speaking $Z$ is a vector field along the injection of $N$ into $M$ ); then any two of the conditions above imply the third.

Although $Z$ need not be tangent to $N$, the conserved quantity $g(Z, \dot{c})$ depends only on its component tangent to $N$.

This result is of course the nonholonomic Noether theorem of [21], for a kinetic energy Lagrangian, in the case where the constraints are actually holonomic! Let us consider the result in this light. The equivalence between the condition $Z^{\mathrm{C}}(L)=0$ on $\mathcal{C}$ and item 2 above is dealt with below. The function $Z^{\mathrm{V}}(L)$ on $\mathcal{C}$ is more-or-less $g(Z, \dot{c})$ (the latter is the former restricted to a base integral curve of $\Gamma)$; note that since $\Gamma$ is tangent to $\mathcal{C}$, in computing $\Gamma\left(Z^{\mathrm{V}}(L)\right)$ we can restrict $Z^{\mathrm{v}}(L)$ to $\mathcal{C}$, i.e. we can set $v^{a}=0$ before acting with $\Gamma$. (The conserved quantity depends only on the component of $Z$ along the constraint distribution, just as we pointed out above for the submanifold case.) The new ingredient is the identification of the condition $\varepsilon(Z)=0$ in terms of the second fundamental form. In fact the definition of the second fundamental form given above extends in a fairly obvious way to a distribution $\mathcal{D}$ (assuming of course one has a metric): for any vector fields $X, Y$ in $\mathcal{D}$ set

$$
\Pi(X, Y)=\frac{1}{2}\left(\nabla_{X} Y+\nabla_{Y} X\right)^{\perp}
$$

where $Z^{\perp}$ is the component of $Z$ perpendicular to $\mathcal{D}$. Note that symmetry is no longer automatic - since we are now dealing with a nonholonomic distribution we won't have $[\eta, \zeta] \in \mathcal{D}$. The condition $\varepsilon(Z)=0$ is just $Z \perp \Pi$.

\subsection{Lagrangians of mechanical type: the general case}

We shall derive expressions for $\varepsilon$ for a Lagrangian of mechanical type,

$$
L(q, u)=\frac{1}{2} g_{q}(u, u)-\phi(q) .
$$

The fibre metric essentially coincides with the metric $g$ on $Q$, at least so far as vertical lifts are concerned. 
First of all, $X^{\mathrm{V}}(L)=g(X, u)$, while $X^{\mathrm{C}}(L)=g\left(\nabla_{u} X, u\right)-X(\phi)$. Let us take a frame $\left\{X_{\alpha}, X_{a}\right\}$ in which the $X_{\alpha}$ span $\mathcal{D}$ and the $X_{a}$ are normal to $\mathcal{D}$, so that $g_{a \alpha}=0$. As before we denote the corresponding quasi-velocities by $\left(v^{\alpha}, v^{a}\right)$, and we denote by $R_{\alpha \beta}^{\gamma} X_{\gamma}$ the component of $\left[X_{\alpha}, X_{\beta}\right]$ in $\mathcal{D}$. We write $\Gamma=v^{\alpha} X_{\alpha}^{\mathrm{C}}+\Gamma^{\alpha} X_{\alpha}^{\mathrm{V}}$. Recall that $\Gamma$ is tangent to $\mathcal{C}$, and that $v^{a}=0$ on $\mathcal{C}$, so that $u=v^{\alpha} X_{\alpha}$ on $\mathcal{C}$, and (for example) $g\left(X_{\alpha}, u\right)=g_{\alpha \beta} v^{\beta}$. Moreover, $v^{\beta} X_{\beta}^{\mathrm{C}}\left(v^{\gamma}\right)=-R_{\beta \delta}^{\gamma} v^{\beta} v^{\delta}=0$ on $\mathcal{C}$ (by skew-symmetry). It follows that

$$
\begin{aligned}
\varepsilon\left(X_{\alpha}\right) & =\left(v^{\beta} X_{\beta}^{\mathrm{C}}+\Gamma^{\beta} X_{\beta}^{\mathrm{V}}\right)\left(g\left(X_{\alpha}, u\right)\right)-g\left(\nabla_{u} X_{\alpha}, u\right)+X_{\alpha}(\phi) \\
& =g_{\alpha \beta} \Gamma^{\beta}+X_{\beta}\left(g_{\alpha \gamma}\right) v^{\beta} v^{\gamma}-g\left(\nabla_{X_{\beta}} X_{\alpha}, X_{\gamma}\right) v^{\beta} v^{\gamma}+X_{\alpha}(\phi) \\
& =g_{\alpha \beta} \Gamma^{\beta}+g\left(X_{\alpha}, \nabla_{X_{\beta}} X_{\gamma}\right) v^{\beta} v^{\gamma}+X_{\alpha}(\phi),
\end{aligned}
$$

from which we can determine $\Gamma$. The calculation of $\varepsilon\left(X_{a}\right)$ is much simplified by the choice of $X_{a}$, since $g\left(X_{a}, u\right)=g\left(X_{a}, v^{\alpha} X_{\alpha}\right)=0$ on $\mathcal{C}$ : thus

$$
\varepsilon\left(X_{a}\right)=-g\left(\nabla_{X_{\alpha}} X_{a}, X_{\beta}\right) v^{\alpha} v^{\beta}+X_{a}(\phi)=g\left(X_{a}, \nabla_{X_{\alpha}} X_{\beta}\right) v^{\alpha} v^{\beta}+X_{a}(\phi) .
$$

In the first term in the final expression, only the component of $\nabla_{X_{\alpha}} X_{\beta}$ normal to $\mathcal{D}$ matters. Let us write $\Pi_{\alpha \beta}=\Pi_{\alpha \beta}^{a} X_{a}$ for the symmetric part of the normal component of $\nabla_{X_{\alpha}} X_{\beta}$ (i.e. the generalized second fundamental form). Then

$$
\varepsilon\left(X_{a}\right)=g_{a b} \Pi_{\alpha \beta}^{b} v^{\alpha} v^{\beta}+X_{a}(\phi),
$$

or more generally, for any vector field $Y$ on $Q$ normal to $\mathcal{D}$,

$$
\varepsilon(Y)=g(Y, \Pi(u, u))+Y(\phi) .
$$

Since $\varepsilon$ annihilates $\mathcal{D}$ and $\Pi$ is normal to it, we conclude that a vector field $Z$ on $Q$ satisfies $\varepsilon(Z)=0$ if and only if $g\left(Z, \Pi_{\alpha \beta}\right)=0$ and $Z^{\perp}(\phi)=0$ where $Z^{\perp}$ is the component of $Z$ normal to $\mathcal{D}$. These are the conditions we alluded to in the discussion on the reaction-annihilator distribution of Section 4.1.

If $g$ is actually a flat metric (as is the case in many examples) then the second fundamental form condition is vacuous.

The conditions for $Z$ to generate a conserved momentum are that

$$
\begin{aligned}
& g\left(Z, \Pi_{\alpha \beta}\right)=0, \quad Z^{\perp}(\phi)=0, \\
& g\left(\nabla_{X_{\alpha}} Z, X_{\beta}\right)+g\left(\nabla_{X_{\beta}} Z, X_{\alpha}\right)=0, \quad Z(\phi)=0 .
\end{aligned}
$$

Regarding the potential $\phi$, note that in fact it isn't enough that $Z(\phi)=0$ : in effect, both the component of $Z$ along $\mathcal{D}$ and the component normal to $\mathcal{D}$ separately have to annihilate $\phi$. The condition $g\left(\nabla_{X_{\alpha}} Z, X_{\beta}\right)+g\left(\nabla_{X_{\beta}} Z, X_{\alpha}\right)=0$ just says that $K_{Z}$, restricted to $\mathcal{D}$, is skew-symmetric. This result may also be found in e.g. [25].

Finally, we make the link to the Riemannian case, as described in Section 5.1. The EulerLagrange field $\Gamma_{0}$ is the geodesic field. Consider a curve $c$ in $Q$, and its natural lift $C=(c, \dot{c})$ to $T Q$. It is easy to see (by a coordinate calculation for example) that the tangent field to the natural lift can be expressed as follows:

$$
\dot{C}=\left.\Gamma_{0}\right|_{C}+\left(\nabla_{\dot{c}} \dot{c}\right)^{\mathrm{V}} .
$$

For a constrained system of this type, therefore, $c$ will be a base integral curve of the constrained dynamical field $\Gamma$ if and only if $\nabla_{\dot{c}} \dot{c}$ is normal to $\left.\mathcal{D}\right|_{c}$. This includes the case in which $\mathcal{D}$ is 
integrable, giving the rule for geodesics in a submanifold. In fact for a base integral curve $c$ of $\Gamma$ we have $\left(\Gamma-\Gamma_{0}\right)_{C}=\left(\nabla_{\dot{c}} \dot{c}\right)^{\mathrm{V}}=\Pi(\dot{c}, \dot{c})^{\mathrm{V}}$. So the formula

$$
\frac{d}{d t}(g(Z, \dot{c}))=K_{Z}(\dot{c}, \dot{c})+g(Z, \Pi(\dot{c}, \dot{c}))
$$

(see above) is the formula

$$
\Gamma\left(Z^{\mathrm{V}}(L)\right)=Z^{\mathrm{C}}(L)+g\left(\Gamma-\Gamma_{0}, Z^{\mathrm{V}}\right)
$$

for this case (along the natural lift $C$ of $c$ ).

\subsection{Quadratic first integrals for constrained systems of mechanical type}

In the previous example we have used the conditions of the nonholonomic Noether theorem as discussed in Section 4. In this section we give an example where we use the more general results of the section on the Cartan form approach. More in particular, we shall apply the conditions of Theorem 1 .

First, a remark about connections. Let $E \rightarrow M$ be a vector bundle with linear connection, with covariant derivative operator $\nabla$ and horizontal lift $X \mapsto X^{\mathrm{H}}$. Any section $\sigma$ of $E \rightarrow M$ determines a vertical vector field on $E$ by the identification of a point of a vector space with a (constant) vector field on it, ie by a variant of the vertical lift construction: call it therefore $\sigma^{\mathrm{v}}$. Then $\left[X^{\mathrm{H}}, \sigma^{\mathrm{V}}\right]=\left(\nabla_{X} \sigma\right)^{\mathrm{V}}$.

Now let $F \rightarrow M$ be a vector sub-bundle of $E$, and suppose that $E$ is equipped with a constant fibre metric $g$. For any $v \in F$, let $\bar{H}_{v}$ be the fibre-orthogonal projection of the horizontal subspace $H_{v}$ of $T_{v} E$ into $T_{v} F$, and $X \mapsto X^{\overline{\mathrm{H}}}$ the corresponding horizontal lift to $F$. Then for any section $\tau$ of $F \rightarrow M,\left[X^{\overline{\mathrm{H}}}, \tau^{\mathrm{V}}\right]$ is a vertical lift, and if we set $\left[X^{\overline{\mathrm{H}}}, \tau^{\mathrm{V}}\right]=\left(\bar{\nabla}_{X} \tau\right)^{\mathrm{V}}$ then $\bar{\nabla}$ is the covariant derivative operator of a linear connection on $F$.

For $x \in M$, let $\left\{e_{i}(x)\right\}$ be a basis for $E_{x}$ such that $\left\{e_{\alpha}(x)\right\}$ is a basis for $F_{x}$ and $\left\{e_{a}(x)\right\}$ a basis for its orthogonal complement. Suppose that we have, locally, such a choice of bases depending smoothly on $x$; in other words, we have local sections $e_{i}$ with these properties. Let $\left\{X_{r}\right\}$ be a local basis of vector fields on $M$, and set $\nabla_{X_{r}} e_{i}=\Gamma_{r i}^{j} e_{j}$; the $\Gamma_{r i}^{j}$ are the connection coefficients of $\nabla$ with respect to the chosen bases. Let $\left(u^{i}\right)$ be the fibre coordinates on $E$ corresponding to the basis of sections $\left\{e_{i}\right\}$, so that $e_{i}^{\mathrm{V}}\left(u^{j}\right)=\delta_{i}^{j}$; note that $F$ is the submanifold $u^{a}=0$. Then

$$
\begin{aligned}
{\left[X_{r}^{\mathrm{H}}, e_{i}^{\mathrm{V}}\right]\left(u^{j}\right) } & =X_{r}^{\mathrm{H}}\left(\delta_{i}^{j}\right)-e_{i}^{\mathrm{V}}\left(X_{r}^{\mathrm{H}}\left(u^{j}\right)\right) \\
& =\left(\nabla_{X_{r}} e_{i}\right)^{\mathrm{V}}\left(u^{j}\right)=\Gamma_{r i}^{j},
\end{aligned}
$$

so that

$$
X_{r}^{\mathrm{H}}\left(u^{j}\right)=-\Gamma_{r i}^{j} u^{i},
$$

as one would expect. Now $X_{r}^{\mathrm{H}}-X_{r}^{\overline{\mathrm{H}}} \in\left\langle e_{a}\right\rangle$, say $X_{r}^{\mathrm{H}}-X_{r}^{\overline{\mathrm{H}}}=\xi_{r}^{a} e_{a}^{\mathrm{V}}$. Thus on $F$ (where $u^{a}=0$ ) $\xi_{r}^{a}=-\Gamma_{r \alpha}^{a} u^{\alpha}$, using the fact that $X_{r}^{\overline{\mathrm{H}}}\left(u^{a}\right)=0$ since $X_{r}^{\overline{\mathrm{H}}}$ is tangent to the submanifold $F$. It follows that $X_{r}^{\overline{\mathrm{H}}}=X_{r}^{\mathrm{H}}+\Gamma_{r \alpha}^{a} u^{\alpha} e_{a}^{\mathrm{V}}$. So finally

$$
\left[X_{r}^{\overline{\mathrm{H}}}, e_{\alpha}^{\mathrm{V}}\right]=\left[X_{r}^{\mathrm{H}}, e_{\alpha}^{\mathrm{V}}\right]+\left[\Gamma_{r \beta}^{a} u^{\beta} e_{a}^{\mathrm{V}}, e_{\alpha}^{\mathrm{V}}\right]=\Gamma_{r \alpha}^{i} e_{i}^{\mathrm{V}}-\Gamma_{r \alpha}^{a} e_{a}^{\mathrm{V}}=\Gamma_{r \alpha}^{\beta} e_{\beta}^{\mathrm{V}} ;
$$

that is to say, the connection coefficients for $\bar{\nabla}$ are $\Gamma_{r \alpha}^{\beta}$. 
Now let us specialize to the case in which $E=T Q$ and $F=\mathcal{C}$, for a constrained system of mechanical type. Choose the basis of vector fields $\left\{X_{\alpha}, X_{a}\right\}$ with $\left\{X_{\alpha}\right\}$ a basis for $\mathcal{D}$ and $X_{a}$ orthogonal to $\mathcal{D}$; then $X_{i}^{\mathrm{V}}$ corresponds to $e_{i}^{\mathrm{V}}$ above. We take for $\nabla$ the Levi-Civita connection of the kinetic energy metric; we have an induced connection $\bar{\nabla}$ on sections of $\mathcal{C}$, i.e. on vector fields in $\mathcal{D}$, with $\bar{\nabla}_{X_{i}} X_{\alpha}=\Gamma_{i \alpha}^{\beta} X_{\beta}$. This is all very much like the definition of the connection induced on a submanifold in Riemannian geometry (the connection $\bar{\nabla}$ may in fact be found in e.g. [29]); note that indeed the generalized second fundamental form is given by

$$
\Pi_{\alpha \beta}^{a}=\frac{1}{2}\left(\Gamma_{\alpha \beta}^{a}+\Gamma_{\beta \alpha}^{a}\right)
$$

Moreover,

$$
\Gamma=v^{\alpha} X_{\alpha}^{\mathrm{C}}-\left(\Gamma_{\beta \gamma}^{\alpha} v^{\beta} v^{\gamma}+(\operatorname{grad} \phi)^{\alpha}\right) X_{\alpha}^{\mathrm{V}}
$$

We have written $\operatorname{grad} \phi$ for the vector field obtained by raising the index on $d \phi$ : that is to say, $g(X, \operatorname{grad} \phi)=X(\phi)$. The corresponding term in $\Gamma$ is (the vertical lift of) the orthogonal projection of $\operatorname{grad} \phi$ onto $\mathcal{D}$. With our present choice of basis this is $g^{\alpha \beta} X_{\beta}(\phi) X_{\alpha}$, where $\left(g^{\alpha \beta}\right)$ is the inverse of $\left(g_{\alpha \beta}\right)$, and also the corresponding block in the inverse of $\left(g_{i j}\right)$. Likewise, the fact that we have $\Gamma_{\beta \gamma}^{\alpha}$ in the first term in the brackets is due to the choice of basis with $X_{a}$ orthogonal to $\mathcal{D}$, and therefore $X_{a}^{\mathrm{V}}$ fibre-normal to $\mathcal{C}$. Now on $\mathcal{C}\left(\right.$ where $\left.v^{a}=0\right)$

$$
\Gamma_{0}=v^{\alpha} X_{\alpha}^{\mathrm{C}}-\left(\Gamma_{\alpha \beta}^{i} v^{\alpha} v^{\beta}+(\operatorname{grad} \phi)^{i}\right) X_{i}^{\mathrm{V}},
$$

so that

$$
\Gamma-\Gamma_{0}=\left(\Pi_{\alpha \beta}^{a} v^{\alpha} v^{\beta}+(\operatorname{grad} \phi)^{a}\right) X_{a}^{\mathrm{V}} .
$$

The vector fields $\left\{X_{\alpha}^{\mathrm{H}}, X_{a}^{\mathrm{H}}, X_{\alpha}^{\mathrm{V}}, X_{a}^{\mathrm{V}}\right\}$ form a local basis for $\mathfrak{X}(T Q)$, of course: denote by $\left\{\theta^{\alpha}, \theta^{a}, \phi^{\alpha}, \phi^{a}\right\}$ the dual basis of 1 -forms. We have

$$
X_{i}^{\overline{\mathrm{H}}}=X_{i}^{\mathrm{H}}+\Gamma_{i \alpha}^{a} v^{\alpha} X_{a}^{\mathrm{V}}
$$

the vector fields $\left\{X_{i}^{\overline{\mathrm{H}}}, X_{\alpha}^{\mathrm{V}}\right\}$ form a basis for $\mathfrak{X}(\mathcal{C})$, with $\left\{X_{\alpha}^{\overline{\mathrm{H}}}, X_{\alpha}^{\mathrm{V}}\right\}$ a basis for $\tilde{\mathcal{D}}$ and $S\left(X_{a}^{\overline{\mathrm{H}}}\right)=X_{a}^{\mathrm{V}}$. Notice that since $\left\langle X_{a}^{\mathrm{V}}, \theta^{\alpha}\right\rangle=\left\langle X_{a}^{\mathrm{V}}, \theta^{b}\right\rangle=\left\langle X_{\alpha}^{\mathrm{V}}, \phi^{\beta}\right\rangle=0,\left\{\iota^{*} \theta^{\alpha}, \iota^{*} \theta^{a}, \iota^{*} \phi^{\alpha}\right\}$ is the dual basis of 1forms on $\mathcal{C}$, and $\left\{\iota^{*} \theta^{a}\right\}$ is a basis for $\tilde{\mathcal{D}}^{\circ}$. We don't claim that these bases correspond exactly to the ones used in Section 3, but nevertheless we can use them to analyse $\iota^{*} \omega_{L}$ in the same way as we did there.

We have

$$
\omega_{L}=g_{\alpha \beta} \phi^{\alpha} \wedge \theta^{\beta}+g_{a b} \phi^{a} \wedge \theta^{b} .
$$

Using the expression for $\omega_{L}$ given above we obtain, on $\mathcal{C}$,

$$
\begin{aligned}
& \left.X_{\alpha}^{\overline{\mathrm{H}}}\right\lrcorner \omega_{L}=-g_{\alpha \beta} \phi^{\beta}+g_{a b} \Gamma_{\alpha \beta}^{a} v^{\beta} \theta^{b} \\
& \left.X_{a}^{\overline{\mathrm{H}}}\right\lrcorner \omega_{L}=-g_{a b} \phi^{b}+g_{b c} \Gamma_{a \alpha}^{b} v^{\alpha} \theta^{c} \\
& \left.X_{\alpha}^{\mathrm{V}}\right\lrcorner \omega_{L}=g_{\alpha \beta} \theta^{\beta} .
\end{aligned}
$$

Then for any vector $Z=\xi^{\alpha} X_{\alpha}^{\overline{\mathrm{H}}}+\xi^{a} X_{a}^{\overline{\mathrm{H}}}+\eta^{\alpha} X_{\alpha}^{\mathrm{V}}$ tangent to $\mathcal{C}$ we find that

$$
\begin{aligned}
& Z\lrcorner \omega_{L}\left(X_{\alpha}^{\overline{\mathrm{H}}}\right)=g_{\alpha \beta} \eta^{\beta}-g_{a b} \Gamma_{\alpha \beta}^{a} v^{\beta} \xi^{b} \\
& Z\lrcorner \omega_{L}\left(X_{a}^{\overline{\mathrm{H}}}\right)=g_{a b} \Gamma_{\alpha \beta}^{b} v^{\beta} \xi^{\alpha}+\left(g_{a c} \Gamma_{b \alpha}^{c}-g_{b c} \Gamma_{a \alpha}^{c}\right) v^{\alpha} \xi^{b} \\
& Z\lrcorner \omega_{L}\left(X_{\alpha}^{\mathrm{V}}\right)=g_{\alpha \beta} \xi^{\beta} .
\end{aligned}
$$


Thus the characteristic vector fields of $\omega_{L}$ take the form $Z=\xi^{a} X_{a}^{\overline{\mathrm{H}}}+\eta^{\alpha} X_{\alpha}^{\mathrm{v}}$ where $\eta^{\alpha}=$ $g^{\alpha \beta} g_{a b} \Gamma_{\beta \gamma}^{a} v^{\gamma} \xi^{b}$, and $\xi^{b}$ satisfies

$$
\left(g_{a c} \Gamma_{b \alpha}^{c}-g_{b c} \Gamma_{a \alpha}^{c}\right) \xi^{b}=0 .
$$

Now the connection coefficients are those for the Levi-Civita connection, but with respect to an anholonomic frame such that $g_{a \alpha}=0$. It follows that $g_{b c} \Gamma_{a \alpha}^{c}+g_{\alpha \beta} \Gamma_{a b}^{\beta}=0$. Thus the condition on $\xi^{a}$ may be written

$$
g_{\alpha \beta}\left(\Gamma_{b a}^{\beta}-\Gamma_{a b}^{\beta}\right) \xi^{b}=0 .
$$

But since the Levi-Civita connection has no torsion, $\left[X_{a}, X_{b}\right]=\left(\Gamma_{a b}^{i}-\Gamma_{b a}^{i}\right) X_{i}$. So the condition on $\xi^{b}$ amounts to $\left[\xi^{a} X_{a}, X_{b}\right] \in \mathcal{D}^{\perp}$, or more generally $\left[\xi^{a} X_{a}, \mathcal{D}^{\perp}\right] \in \mathcal{D}^{\perp}$. That is, $\xi^{a} X_{a}$ (a vector field in $\mathcal{D}^{\perp}$ ) has the property that its bracket with every vector field in $\mathcal{D}^{\perp}$ belongs to $\mathcal{D}^{\perp}$. This condition determines a subdistribution of $\mathcal{D}^{\perp}$, which is easily seen to be integrable (by the Jacobi identity). It's obviously a fundamental feature of $\mathcal{D}^{\perp}$ (or indeed of any distribution). It is perfectly possible, if the dimensions are right, for this distribution to reduce to the zero vector, in which case $\iota^{*} \omega_{L}$ is symplectic: this should be an interesting class of constrained systems. The so-called nonholonomic particle in 3 dimensions is an example.

Now $\mathcal{C}$ is a vector bundle, and so has associated with it tensor bundles of all types, which we shall call $\mathcal{C}$-tensor bundles. Let $A$ be a symmetric type $(0,2) \mathcal{C}$-tensor. With respect to the local basis of sections $\left\{X_{\alpha}\right\}$ of $\mathcal{C}, A$ has components $A_{\alpha \beta}$; we can think of $A$ as the corresponding collection of functions on $Q$, with the transformation rule induced by that of a change of basis for $\mathcal{D}$. Then $A_{\alpha \beta} v^{\alpha} v^{\beta}$ is a well-defined function on $\mathcal{C}$. Let $f$ be a function on $Q$. We seek the conditions for $\psi=A_{\alpha \beta} v^{\alpha} v^{\beta}+f$ to be a conserved quantity for $\Gamma$. The vector field $Z_{\psi} \in \tilde{\mathcal{D}}$ from Theorem 1 determined by $\psi$ is easily seen to be given by

$$
g^{\alpha \beta}\left(X_{\alpha}^{\overline{\mathrm{H}}}(\psi) X_{\beta}^{\mathrm{V}}-X_{\alpha}^{\mathrm{V}}(\psi) X_{\beta}^{\overline{\mathrm{H}}}\right),
$$

so $\psi$ is conserved if and only if

$$
g^{\alpha \beta}\left(X_{\alpha}^{\overline{\mathrm{H}}}(\psi) X_{\beta}^{\mathrm{V}}\left(E_{L}\right)-X_{\alpha}^{\mathrm{V}}(\psi) X_{\beta}^{\overline{\mathrm{H}}}\left(E_{L}\right)\right)=0 .
$$

In the computation it helps to note that we can replace $X_{\alpha}^{\overline{\mathrm{H}}}$ by $X_{\alpha}^{\mathrm{H}}$ since there are no occurrences of $v^{a} ; X_{\alpha}^{\mathrm{H}}\left(v^{\beta}\right)=-\Gamma_{\alpha \gamma}^{\beta} v^{\gamma}$ on $\mathcal{C}$; and $X_{\alpha}^{\mathrm{H}}(T)=0$ where $T$ is the kinetic energy. We obtain

$$
\left(X_{\alpha}\left(A_{\beta \gamma}\right)-A_{\delta \gamma} \Gamma_{\alpha \beta}^{\delta}-A_{\beta \delta} \Gamma_{\alpha \gamma}^{\delta}\right) v^{\alpha} v^{\beta} v^{\gamma}+\left(X_{\alpha}(f)-2 A_{\alpha \beta}(\operatorname{grad} \phi)^{\beta}\right) v^{\alpha}=0 .
$$

So the necessary and sufficient conditions for $\psi=A_{\beta \gamma} v^{\beta} v^{\gamma}+f$ to be a constant are that

$$
\bar{\nabla}_{(\alpha} A_{\beta \gamma)}=0, \quad X_{\alpha}(f)=2 A_{\alpha \beta}(\operatorname{grad} \phi)^{\beta} .
$$

(We have used brackets around indices to denote symmetrization, so that for example $T_{(i j k)}$ are the components of the symmetrized tensor derived from a tensor $T$, ie $\frac{1}{6} \sum T_{i j k}$ where the sum is over all permutations of $i, j$ and $k$. In view of the assumption that $A_{\alpha \beta}$ is symmetric in its indices, $\bar{\nabla}_{(\alpha} A_{\beta \gamma)}$ is in this case a constant multiple of the cyclic sum.)

This is a direct analogue of the corresponding result for the unconstrained system (see [10]). That this is so is due to the fact that we are working with an anholonomic frame, to the choice of frame, and to the use of the induced connection $\bar{\nabla}$.

The next question is what happens if $A$ is actually the restriction of a tensor on $Q$ ? The answer is easier to state if we assume that the tensor on $Q$ is also symmetric, but this is not essential - 
all we require is that the restriction to $\mathcal{D}$ is symmetric. On the other hand, there is no significant loss of generality in working with symmetric tensors. So let $A$ be a symmetric type $(0,2)$ tensor on $Q$. For the restriction to $\mathcal{C}$ of the function $A_{i j} v^{i} v^{j}+f$ to be a conserved quantity for $\Gamma$ the components $A_{\alpha \beta}$ of $A$ must satisfy the conditions above. Since $A$ is a tensor on $Q$ we can attempt to express those conditions in terms of $\nabla$. Then the condition $\bar{\nabla}_{(\alpha} A_{\beta \gamma)}=0$ becomes

$$
\nabla_{(\alpha} A_{\beta \gamma)}+A_{a(\alpha} \Pi_{\beta \gamma)}^{a}=0
$$

We are not forced to take $A_{a(\alpha} \Pi_{\beta \gamma)}^{a}=0$, though it is obviously convenient if it holds. The condition $X_{\alpha}(f)=2 A_{\alpha \beta}(\operatorname{grad} \phi)^{\beta}$ is unchanged.

Finally, suppose that $A_{i j} v^{i} v^{j}+f$ is a conserved quantity for the unconstrained system. Under what conditions is it also a conserved quantity for the constrained system? From the assumption that it is conserved for the unconstrained system we have

$$
\nabla_{(i} A_{j k)}=0, \quad X_{i}(f)=2 A_{i j}(\operatorname{grad} \phi)^{j} .
$$

From the first, in particular $\nabla_{(\alpha} A_{\beta \gamma)}=0$, so we require in addition that $A_{a(\alpha} \Pi_{\beta \gamma)}^{a}=0$. From the second, $X_{\alpha}(f)=2 A_{\alpha \beta}(\operatorname{grad} \phi)^{\beta}+2 A_{\alpha a}(\operatorname{grad} \phi)^{a}$, so we require in addition that $A_{\alpha a}(\operatorname{grad} \phi)^{a}=$ 0 .

These results include and extend those of Iliev and Semerdzhiev [24, 25] on quadratic integrals. It is easy to extend them also to constants of higher degree in $v$; however, this is the most interesting case because of the interaction between $f$ and $\phi$. For higher degree constants of the form $A_{\alpha \beta \ldots \delta} v^{\alpha} v^{\beta} \cdots v^{\delta}+f$ the conditions corresponding to the first set are

$$
\bar{\nabla}_{(\alpha} A_{\beta \gamma \ldots)}=0, \quad X_{\alpha}(f)=0, \quad A_{\alpha \beta \ldots \delta}(\operatorname{grad} \phi)^{\delta}=0 .
$$

\section{Acknowledgements}

The first author is a Guest Professor at Ghent University: he is grateful to the Department of Mathematics for its hospitality. The second author is a Postdoctoral Fellow of the Research Foundation - Flanders (FWO). This work is part of the IRSES project GEOMECH (nr. 246981) within the 7th European Community Framework Programme. We are indebted to W. Sarlet for many useful discussions.

\section{References}

[1] L. Bates and J. Śniatycki, Nonholonomic reduction, Rep. Math. Phys. 32 (1993) 99-115.

[2] A. M. Bloch with the collaboration of J. Baillieul, P. Crouch and J. E. Marsden, Nonholonomic Mechanics and Control (Springer, 2003).

[3] A. M. Bloch, P. S. Krishnaprasad, J. E. Marsden and R. M. Murray, Nonholonomic mechanical systems with symmetry, Arch. Rational Mech. Anal. 136 (1996) 21-99.

[4] A. M. Bloch, J. E. Marsden and D. V. Zenkov, Quasi-velocities and symmetries in nonholonomic systems, Dynamical Systems 24 (2009) 187-222.

[5] F. Cantrijn, J. Cortés, M. de León and D. Martín de Diego, On the geometry of generalized Chaplygin systems, Math. Proc. Camb. Phil. Soc. 132 (2002) 323-351. 
[6] H. Cendra, J.E. Marsden and T.S. Ratiu, Geometric mechanics, Lagrangian reduction, and nonholonomic systems, in Mathematics Unlimited - 2001 and Beyond, eds. B. Engquist and W. Schmid (Springer, 2001) pp. 221-273.

[7] J. Cortés Monforte, Geometric, Control and Numerical Aspects of Nonholonomic Systems (Lecture Notes in Mathematics 1793, Springer, 2002).

[8] J. Cortés and M. de León, Reduction and reconstruction of the dynamics of nonholonomic systems, J. Phys. A 32 (1999) 8615-8645.

[9] M. Crampin, Constants of the motion in Lagrangian mechanics, Int. J. Theor. Phys. 16 (1977) $741-754$.

[10] M. Crampin, Hidden symmetries and Killing tensors, Rep. Math. Phys. 20 (1984) 31-40.

[11] M. Crampin and T. Mestdag, Anholonomic frames in constrained dynamics, Dynamical Systems 25 (2010) 159-187.

[12] M. Crampin and T. Mestdag, Reduction of invariant constrained systems using anholonomic frames, math.DG/1101.2551.

[13] M. Crampin and F. A. E. Pirani, Applicable Differential Geometry (LMS Lecture Notes 59, Cambridge University Press, 1988).

[14] R.H. Cushman, H. Duistermaat and J. Śniatycki, Geometry of Nonholonomically Constrained Systems (Advanced Series in Nonlinear Dynamics 26, World Scientific, 2010).

[15] R. Cushman, D. Kemppainen, J. Śniatycki and L. Bates, Geometry of nonholonomic constraints, Rep. Math. Phys. 36 (1995) 275-286.

[16] M. de León and D. Martin de Diego, On the geometry of non-holonomic Lagrangian systems, J. Math. Phys. 37 (1996) 3389-3414.

[17] M. de León and P. R. Rodrigues, Methods of Differential Geometry in Analytical Mechanics (North-Holland Math. Studies 158, Elsevier, 1989).

[18] K. Ehlers, J. Koiller, R. Montgomery and P. M. Rios, Nonholonomic systems via moving frames: Cartan equivalence and Chaplygin Hamiltonization, The Breadth of Symplectic Geometry, eds. J. E. Marsden et al. (Birkhäuser, 2005) pp. 75-116.

[19] F. Fassò, A. Giacobbe and N. Sansonetto, Gauge conservation laws and the momentum equation in nonholonomic mechanics, Rep. Math. Phys. 62 (2008) 345-367.

[20] F. Fassò, A. Giacobbe and N. Sansonetto, On the number of weakly Noetherian constants of motion of nonholonomic systems, J. Geom. Mech. 1 (2009) 389-416.

[21] F. Fassò, A. Ramos and N. Sansonetto, The reaction-annihilator distribution and the nonholonomic Noether theorem for lifted actions, Regul. Chaotic Dyn. 12 (2007) 579-588.

[22] F. Fassò and N. Sansonetto, An elemental overview of the nonholonomic Noether theorem, Int. J. Geom. Methods Mod. Phys. 6 (2009) 1343-1355.

[23] G. Giachetta, First integrals of non-holonomic systems and their generators, J. Phys. A: Math. Gen. 33 (2000) 5369-5389. 
[24] Il. Iliev, On first integrals of a nonholonomic mechanical system, J. Appl. Math. Mech. 39 (1975) 147-150.

[25] Il. Iliev and Khr. Semerdzdhiev, Relations between first integrals of a nonholonomic mechanical system and of the corresponding system freed of constraints, J. Appl. Math. Mech. 36 (1972) 381-388.

[26] J. Koiller, Reduction of some classical non-holonomic systems with symmetry, Arch. Rat. Mech. Anal. 118 (1992) 113-148.

[27] Y. Kosman-Schwarzbach, The Noether Theorems. Invariance and Conservation Laws in the Twentieth Century (Springer, 2011).

[28] D. Krupka, O. Krupková and D. Saunders, The Cartan form and its generalizations in the calculus of variations, Int. J. Geom. Methods Mod. Phys. 7 (2010) 631-654.

[29] A. D. Lewis, Affine connections and distributions with applications to nonholonomic mechanics, Rep. Math. Phys. 42 (1998), 135-164.

[30] G. Marmo, G. Morandi, A. Simoni and E. C. G Sudarshan, Quasi-invariance and central extensions, Phys. Rev. D 37 (1988) 2196-2205.

[31] R. Montgomery, A Tour of Subriemannian Geometries, their Geodesics and Applications (AMS, 2002).

[32] W. Sarlet and F. Cantrijn, Generalizations of Noether's theorem in classical mechanics, SIAM Review 23 (1981) 467-494.

[33] D. V. Zenkov, Linear conservation laws of nonholonomic systems with symmetry, Proceedings of the Fourth International Conference on Dynamical Systems and Differential Equations, Wilmington, NC, 2002, pp. 967-976. 